ACCepted to ApJ: March 18, 2011

Preprint typeset using $\mathrm{LAT}_{\mathrm{E}} \mathrm{X}$ style emulateapj v. 11/10/09

\title{
GLOBAL SELF-SIMILAR PROTOSTELLAR DISK/WIND MODELS
}

\author{
Seth Teither ${ }^{1,2}$ \\ Department of Astronomy \& Astrophysics, University of Chicago, 5640 S Ellis Ave, Chicago, IL 60637, USA \\ ACCePted to ApJ: March 18, 2011
}

\begin{abstract}
The magnetocentrifugal disk wind mechanism is the leading candidate for producing the large-scale, bipolar jets commonly seen in protostellar systems. I present a detailed formulation of a global, radially self-similar model for a non-ideal disk that launches a magnetocentrifugal wind. This formulation generalizes the conductivity tensor formalism previously used in radially localized disk models. The model involves matching a solution of the equations of non-ideal MHD describing matter in the disk to a solution of the equations of ideal MHD describing a "cold" wind. The disk solution must pass smoothly through the sonic point, the wind solution must pass smoothly through the Alfvén point, and the two solutions must match at the disk/wind interface. This model includes for the first time a self-consistent treatment of the evolution of magnetic flux threading the disk, which can change on the disk accretion timescale. The formulation presented here also allows a realistic conductivity profile for the disk to be used in a global disk/wind model for the first time. The physical constraints on the model solutions fix the distribution of the magnetic field threading the disk, the midplane accretion speed, and the midplane migration speed of flux surfaces. I present a representative solution that corresponds to a disk in the ambipolar conductivity regime with a nominal neutral-matter-magneticfield coupling parameter that is constant along field lines, matched to a wind solution. I conclude with a brief discussion of the importance of self-similar disk/wind models in studying global processes such as dust evolution in protostellar systems.
\end{abstract}

Subject headings: accretion, accretion disks - ISM: jets and outflows - magnetohydrodynamics - stars: formation - stars: protostars - stars: winds, outflows

\section{INTRODUCTION}

Low-mass star formation typically involves the collapse of a dense core inside a molecular cloud to form a condensed central object and an extended disk. The protostar gains much of its mass by accretion of disk material, which can only proceed if there is some mechanism for removing angular momentum from the accreting material. Protostars that are actively accreting disk material frequently exhibit associated bipolar outflows in the form of collimated jets oriented perpendicular to the disk, stretching out over distances much larger than the radial scale of the disk (e.g., Bally et al. 2007). This association between accretion disks and large-scale, collimated jets is also seen in other systems, including Xray binaries and active galactic nuclei. The strongest candidate mechanism for launching jets in these systems is described by the magnetocentrifugal disk wind model (Blandford \& Payne 1982, hereafter BP82), which relies on the acceleration of a fraction of disk material out along a large-scale, ordered magnetic field threading the disk at a sufficiently small inclination angle. This magnetic field could be the interstellar field in the parent molecular cloud that is dragged in during core collapse, or it could be generated by dynamo activity in the disk or the star. A natural consequence of the disk wind model is that the wind efficiently removes angular momentum

\footnotetext{
${ }^{1}$ Presented as a dissertation to the Department of Astronomy and Astrophysics, The University of Chicago, in partial fulfillment of the requirements for the Ph.D. degree.

${ }^{2}$ Current address: Department of Astronomy, University of Wisconsin-Madison, 475 N. Charter St., Madison, WI 53706 , USA; email: steitler@astro.wisc.edu
}

from the disk material, which could explain the strong inferred link between accretion and outflow phenomena in protostars (e.g., Königl \& Salmeron 2011).

Several previous semianalytic studies have matched solutions of the equations of non-ideal MHD that describe a weakly ionized, magnetized accretion disk, and solutions of the equations of cold, ideal MHD for a polytropic fluid that describe a BP82-type magnetocentrifugal wind that removes all the disk's excess angular momentum. These models focused on a radially localized portion of the disk (e.g., Wardle \& Königl 1993, hereafter WK93; Salmeron et al. 2007, hereafter SKW07; Salmeron et al. 2011, hereafter SKW11), or on a global disk model (e.g., Königl 1989; Li 1996, hereafter L96; Ferreira 1997, hereafter F97) based on the same assumption of self-similarity in spherical radius that underlies the BP82 wind model. Another approach is presented in Campbell (2003, 2005), where the thinness of the disk is used to justify solutions for the disk structure that are separable in the radial and vertical coordinates; these solutions are matched to an isothermal wind. A key parameter in determining whether a disk can launch a magnetocentrifugal wind is the field line inclination angle at the disk surface $B_{r, \mathrm{~s}} / B_{z, \mathrm{~s}}$, where $B_{r, \mathrm{~s}}$ and $B_{z, \mathrm{~s}}$ are the radial and vertical field components at the disk surface (subscript 's'). This inclination angle is determined by the flux distribution along the disk surface and can only be calculated in a self-consistent fashion in a global treatment (Ogilvie \& Livio 2001, hereafter OL01). A second key parameter describing the interaction of the disk and wind is $B_{\phi, s}$, the azimuthal field strength at the disk surface, which sets the torque exerted by the wind on the 
disk (BP82). As with the surface field inclination angle, the value of $B_{\phi, \mathrm{s}}$ is determined by processes acting outside the disk; specifically, the value of $B_{\phi, \mathrm{s}}$ is determined by the constraint that the wind solution pass smoothly through the Alfvén point.

Accretion flows in a weakly ionized disk will drag in magnetic flux, opposing the natural tendency of the magnetic flux to diffuse outwards. Studies of accretion disks that shed angular momentum due to MHD turbulence developed from the magnetorotational instability (MRI, Balbus \& Hawlev 1991) suggest that the magnetic diffusivity and the effective viscosity of such disks are comparable (Guan \& Gammie 2009; Lesur \& Longaretti 2009), agreeing with previous work by Lubow et al. (1994a) which suggested that the magnetic diffusivity of MRIactive accretion disks may be too large to allow efficient inward dragging of magnetic flux. This problem can be overcome in an MRI-active disk threaded by a large-scale field that removes angular momentum from surface layers in which the MRI is suppressed (Rothstein \& Lovelace 2008; Lovelace et al. 2009). More generally, this problem disappears if the angular momentum transport mechanism is decoupled from the mechanism that provides the magnetic diffusivity so that the diffusivity is much smaller than the effective viscosity of the disk. This occurs naturally in a disk from which angular momentum is removed through a magnetocentrifugal wind, allowing magnetic flux to be dragged in and distributed over a wind-driving disk. As noted by OL01, the evolution of the magnetic flux through the disk determines whether magnetic configurations capable of launching outflows can be maintained over the accretion timescale. Previous studies of wind-driving disks have not treated the effects of magnetic flux evolution in a self-consistent manner: in a local model, OL01 calculated the rate of flux migration given $B_{r, \mathrm{~s}}$ and $B_{\phi, \mathrm{s}}$, which were free parameters. The local models of Campbell (2003, 2005) expressed $B_{\phi, \mathrm{s}}$ and the surface field inclination angle in terms of unconstrained parameters that describe the structure of the disk and the processes operating within it. WK93 also employed a local model in which they determined $B_{\phi, \mathrm{s}}$ self-consistently using the Alfvén point constraint, but left the surface field inclination angle unconstrained, describing the magnetic flux migration speed using the free parameter $\epsilon_{\mathrm{B}}$. The global models of L96 and F97 also solved for $B_{\phi, \mathrm{s}}$ using the Alfvén point constraint but left the surface field inclination angle and magnetic flux migration unconstrained, essentially setting $\epsilon_{\mathrm{B}}=0$. For an overview of recent work on semianalytic models and complementary numerical simulations of protostellar jets, see the article by Königl \& Salmeron (2011).

I present here a new, semianalytic, radially self-similar, global model that matches disk solutions with BP82-type wind solutions, adopting an approach similar to that of L96 but including for the first time a fully self-consistent calculation of the effects of magnetic flux migration and the magnetic field structure above the disk: $\epsilon_{\mathrm{B}}, B_{r, \mathrm{~s}}$, $B_{\phi, \mathrm{s}}$ and $B_{r, \mathrm{~s}} / B_{z, \mathrm{~s}}$ are no longer free parameters but are determined by physical constraints imposed at the disk surface and at critical points of the outflow. In addition, the equations describing the disk are formulated in terms of the conductivity tensor, allowing for fully general non-ideal disk models. The plan of the paper is as follows. In Section $2 \mathrm{I}$ review the equations describing a (nearly) steady-state, axisymmetric, weakly ionized disk threaded by a magnetic field, and I show how the system reduces to a set of ordinary differential equations under the radial self-similarity assumption. In Section 3 I discuss constraints from the self-consistent treatment of the field-line inclination at the top of the disk and from matching disk and wind solutions. In Section 4 I present a representative matched disk/wind solution, and in Section 5 I summarize the study and outline plans to improve on the current model.

\section{FORMULATION}

I consider a weakly ionized, geometrically thin, vertically isothermal, axisymmetric disk in near-Keplerian rotation around a protostar of mass $M$ that is threaded by an ordered, large-scale magnetic field. I examine the general, non-ideal MHD case (Section 2.1), expressing the matter-field interaction using the conductivity tensor formalism, and seek solutions that are steady on timescales shorter than the accretion time (Section 2.1.1). I follow L96 and F97 in making the simplifying assumption of radial self-similarity to produce a global disk/wind model (Section 2.1.2), and I derive the midplane values of various physical quantities (Section 2.1.3).

In Section 2.2 I specialize to the case of the ambipolar conductivity regime. I once again derive the relevant selfsimilar disk equations (Section 2.2.2) and the midplane values for the variables of integration (Section 2.2.3).

\subsection{General Non-Ideal Case}

The interiors of protostellar disks have a low ionization fraction, necessitating the use of non-ideal MHD to describe the behavior of the disk material in the presence of a large-scale magnetic field that is assumed to thread the disk. The non-ideal matter-field interaction can be described using a multi-fluid approach or by introducing the conductivity tensor (e.g., Königl \& Salmeron 2011), which encapsulates the response of the dominant neutral fluid to the presence of the magnetic field. The effects of the magnetic field are communicated to the neutrals by the development of charge-neutral drift velocities due to the Lorentz forces on charged species, and the consequent systematic charge-neutral drag forces. Under the conductivity tensor formalism, this is expressed in the generalized version of Ohm's law,

$$
\boldsymbol{J}=\boldsymbol{\sigma} \cdot \boldsymbol{E}_{\mathrm{c}}=\sigma_{\mathrm{O}} \boldsymbol{E}_{\mathrm{c} \|}+\sigma_{\mathrm{H}} \hat{\boldsymbol{B}} \times \boldsymbol{E}_{\mathrm{c} \perp}+\sigma_{\mathrm{P}} \boldsymbol{E}_{\mathrm{c} \perp},
$$

where $\boldsymbol{E}_{\mathrm{c}}$ is the electric field in a frame comoving with the dominant neutral fluid, $\boldsymbol{E}_{\mathrm{c} \|}$ and $\boldsymbol{E}_{\mathrm{c} \perp}$ are the components of $\boldsymbol{E}_{\mathrm{c}}$ parallel and perpendicular to the magnetic field, respectively, and $\boldsymbol{\sigma}$ is the conductivity tensor. The components of $\boldsymbol{\sigma}$ are the Ohm conductivity $\sigma_{\mathrm{O}}$, the Hall conductivity $\sigma_{\mathrm{H}}$, and the Pedersen conductivity $\sigma_{\mathrm{P}}$.

For a given charged species (denoted by subscript ' $j$ '), the product of the gyrofrequency $\left(\frac{\left|Z_{j}\right| e|B|}{m_{j} c}\right)$ and the neutral-charge momentum exchange time $1 /\left(\gamma_{j} \rho\right)$ gives a measure of the coupling of the charged species to the magnetic field in a sea of neutrals. In these expressions, $Z_{j}, m_{j}$, and $\gamma_{j}$ are the signed charge, mass, and chargeneutral collisional drag coefficient of species $j$, and $\rho$ is the neutral mass density. The signed value of the ratio of 
the gyrofrequency and neutral collision frequency is the Hall parameter,

$$
\beta_{j} \equiv \frac{\left|Z_{j}\right| e B}{m_{j} c} \frac{1}{\gamma_{j} \rho} .
$$

The definition of the Hall parameter uses the signed value of the magnetic field strength, $B \equiv|\boldsymbol{B}| \operatorname{sgn}\left\{B_{z}\right\}$, where $B_{z}$ is the $z$ component of the magnetic field in cylindrical coordinates $\{r, \phi, z\}$, so that the Hall conductivity retains its dependence on the magnetic field polarity (see Equation (4)). Large values $|\beta|_{j} \gg 1$ imply that charged species $j$ is tightly coupled to the magnetic field, whereas small values $\left|\beta_{j}\right| \ll 1$ imply that species $j$ is weakly coupled to the field.

The conductivity tensor components can be expressed in terms of the Hall parameters as

$$
\begin{gathered}
\sigma_{\mathrm{O}}=\frac{e c}{B} \sum_{j} n_{j}\left|Z_{j}\right| \beta_{j}, \\
\sigma_{\mathrm{H}}=\frac{e c}{B} \sum_{j} \frac{n_{j}\left|Z_{j}\right|}{1+\beta_{j}^{2}},
\end{gathered}
$$

and

$$
\sigma_{\mathrm{P}}=\frac{e c}{B} \sum_{j} \frac{n_{j}\left|Z_{j}\right| \beta_{j}}{1+\beta_{j}^{2}}
$$

Note that since $B$ is the signed value of the magnetic field strength, the Hall conductivity can be positive or negative. The factor of $B$ in $\beta_{j}$ cancels the pre-factor of $1 / B$ in the expressions for the Ohm and Pedersen conductivities, which are positive regardless of the magnetic field orientation.

The coupling of the neutral matter to the magnetic field is described by the Elsasser number $\Lambda$, given by

$$
\Lambda \equiv \frac{v_{\mathrm{A}}^{2}}{\Omega_{\mathrm{K}} \eta_{\perp}},
$$

where $v_{\mathrm{A}} \equiv|\boldsymbol{B}| / \sqrt{4 \pi \rho}$ is the Alfvén speed, $\Omega_{\mathrm{K}}$ is the Keplerian angular frequency, $\eta_{\perp}=c^{2} / 4 \pi \sigma_{\perp}$, and $\sigma_{\perp}=$ $\left(\sigma_{\mathrm{H}}^{2}+\sigma_{\mathrm{P}}^{2}\right)^{1 / 2}$ (e.g., Königl \& Salmeron 2011). As with the Hall parameter, large values $\Lambda \gg 1$ imply that the neutral matter is strongly coupled to the magnetic field, whereas small values $\Lambda \ll 1$ imply that the neutral matter is only weakly coupled to the magnetic field. Launching a magnetocentrifugal wind typically requires strong neutral-field coupling $(\Lambda \gtrsim 1)$.

\subsubsection{Governing Equations}

The governing equations for the disk are the continuity of mass,

$$
\nabla \cdot(\rho \boldsymbol{V})=0
$$

and the conservation of momentum,

$$
\frac{\partial \boldsymbol{V}}{\partial t}+(\boldsymbol{V} \cdot \nabla) \boldsymbol{V}+\frac{1}{\rho} \nabla P+\nabla \Phi-\frac{\boldsymbol{J} \times \boldsymbol{B}}{c \rho}=0 .
$$

In these equations, $\boldsymbol{V}$ is the neutral gas velocity and $P=$ $\rho c_{\mathrm{s}}^{2}$ is its pressure, where $c_{\mathrm{s}}$ is the isothermal speed of sound. The gravitational potential of the central object is given by $\Phi=-G M / R=-V_{\mathrm{K}}^{2}(R)$, where $R=\left(r^{2}+\right.$ $\left.z^{2}\right)^{1 / 2}$ is the spherical radius and $V_{\mathrm{K}}$ is the Keplerian rotation speed.

Within the disk, the ionization fraction is very low, which implies that the equations of momentum conservation for the charged species are dominated by the chargeneutral drag force and the Lorentz force (cf. Equations 2.15-2.17 in WK93). We can thus replace the chargeneutral drag force with the Lorentz force; this substitution has already been made in the conservation of momentum for the neutral disk material (Equation (8)).

The current density satisfies Ampère's law (neglecting the displacement current)

$$
\boldsymbol{J}=\frac{c}{4 \pi} \nabla \times \boldsymbol{B},
$$

and Ohm's law (Equation (1)), with the comoving electric field $\boldsymbol{E}_{\mathrm{c}}$ related to the inertial-frame electric field Eby

$$
\boldsymbol{E}_{\mathrm{c}}=\boldsymbol{E}+\frac{\boldsymbol{V} \times \boldsymbol{B}}{c} .
$$

The magnetic field satisfies the solenoidal condition

$$
\nabla \cdot \boldsymbol{B}=0,
$$

and the electric field $\boldsymbol{E}$ satisfies the induction equation,

$$
\frac{\partial \boldsymbol{B}}{\partial t}=-c \nabla \times \boldsymbol{E},
$$

or, in terms of the comoving electric field,

$$
\frac{\partial \boldsymbol{B}}{\partial t}=\nabla \times(\boldsymbol{V} \times \boldsymbol{B})-c \nabla \times \boldsymbol{E}_{\mathrm{c}} .
$$

In a strictly steady state, $\partial \boldsymbol{B} / \partial t=0$ identically. However, the magnetic flux threading the disk interior to a fixed radius $r$ can change on the accretion timescale due to advection of field lines by the accretion flow and diffusion of field lines through the non-ideal disk material. I therefore consider disk solutions that are steady on timescales longer than the dynamic time $\sim r / V_{\mathrm{K}}$ but relax the steady-state assumption on timescales comparable to the accretion time $\sim r /\left|V_{r}\right|$.

Expanding Equation (13) yields

$$
\begin{gathered}
\frac{\partial B_{r}}{\partial t}=V_{r} \frac{\partial B_{z}}{\partial z}+B_{z} \frac{\partial V_{r}}{\partial z}-V_{z} \frac{\partial B_{r}}{\partial z} \\
-B_{r} \frac{\partial V_{z}}{\partial z}+c \frac{\partial E_{\mathrm{c} \phi}}{\partial z} \\
\frac{\partial B_{\phi}}{\partial t}=B_{r} \frac{\partial V_{\phi}}{\partial r}+B_{z} \frac{\partial V_{\phi}}{\partial z}-\frac{V_{\phi} B_{r}}{r}-V_{z} \frac{\partial B_{\phi}}{\partial z}-B_{\phi} \frac{\partial V_{z}}{\partial z} \\
-B_{\phi} \frac{\partial V_{r}}{\partial r}-V_{r} \frac{\partial B_{\phi}}{\partial r}+c \frac{\partial E_{\mathrm{c} z}}{\partial r}-c \frac{\partial E_{\mathrm{c} r}}{\partial z}
\end{gathered}
$$

and

$$
\begin{aligned}
\frac{\partial B_{z}}{\partial t}= & \frac{V_{z} B_{r}}{r}-\frac{V_{r} B_{z}}{r}+V_{z} \frac{\partial B_{r}}{\partial r}+B_{r} \frac{\partial V_{z}}{\partial r} \\
& -V_{r} \frac{\partial B_{z}}{\partial r}-B_{z} \frac{\partial V_{r}}{\partial r}-c \frac{E_{\mathrm{c} \phi}}{r}-c \frac{\partial E_{\mathrm{c} \phi}}{\partial r},
\end{aligned}
$$

where the solenoidal condition (Equation (111) was used to obtain Equation (15). Now, $V_{\phi} \approx V_{\mathrm{K}},\left|V_{r}\right| \approx c_{\mathrm{s}}$ through much of the disk and $\left|V_{r}\right| \approx V_{z}$ near the top of the disk (see Figure 2), $z / r \lesssim h_{\mathrm{T}} / r=c_{\mathrm{s}} / V_{\mathrm{K}} \ll 1$ (where 
$h_{\mathrm{T}}$ is the tidal scale height), and $c \boldsymbol{E}_{\mathrm{c}} \approx \boldsymbol{V} \times \boldsymbol{B}$. These relations imply that the terms $V_{r} \partial B_{z} / \partial z, B_{z} \partial V_{r} / \partial z$, $V_{z} \partial B_{r} / \partial z, B_{r} \partial V_{z} / \partial z$, and $c \partial E_{\mathrm{c} \phi} / \partial z$ in Equation (14) describe processes operating on the dynamic timescale. Similarly, the terms $B_{r} \partial V_{\phi} / \partial r, B_{z} \partial V_{\phi} / \partial z, V_{\phi} B_{r} / r$, $V_{z} \partial B_{\phi} / \partial z, B_{\phi} \partial V_{z} / \partial z, c \partial E_{\mathrm{c} z} / \partial r$, and $c \partial E_{\mathrm{c} r} / \partial z$ in Equation (15) are associated with processes operating on the dynamic timescale. However, every term in Equation (16) describes a process that operates on the longer accretion timescale. I therefore ignore $\partial B_{r} / \partial t$ and $\partial B_{\phi} / \partial t$ in the $r$ and $\phi$ components of the induction equation, since those terms represent changes in the magnetic field on the accretion timescale and are negligible compared with the terms representing changes in the magnetic field on the dynamic timescale, but I retain $\partial B_{z} / \partial t$ in the $z$ component of the induction equation (cf. Königl et al. 2010)

The components of Equation (12) thus become

$$
\begin{gathered}
c \frac{\partial E_{\phi}}{\partial z}=0 \\
c \frac{\partial E_{r}}{\partial z}-c \frac{\partial E_{z}}{\partial r}=0
\end{gathered}
$$

and

$$
\frac{\partial B_{z}}{\partial t}=\frac{c}{r} \frac{\partial\left(r E_{\phi}\right)}{\partial r} .
$$

The $r$ and $z$ components of the induction equation together imply that $E_{\phi}$ is constant with height at a given $r$ but need not vanish identically.

\subsubsection{Self-Similarity Equations}

The disk equations reduce from partial to ordinary differential equations under the assumption of radial selfsimilarity, which implies that all quantities are described by power laws in the spherical radius $R$ for a fixed inclination angle. I normalize all quantities by midplane values on a reference magnetic field line (subscript ' $*$ '), using a scheme similar to that found in $\mathrm{Li}(1995)$ and L96. Magnetic field lines can be labeled by the magnetic flux function $\Psi$, which satisfies $\nabla \Psi \times \hat{\phi}=B_{\mathrm{p}} r$, where $B_{\mathrm{p}}$ is the poloidal magnetic field. Note that the notation used here corresponds to that of $\mathrm{Li}(1995)$ and L96 but differs from that of Königl et al. (2010), where the flux function is labeled $A$ and $\Psi$ is used to denote the poloidal flux; the two quantities are related by $A=\Psi / 2 \pi$.

Under the self-similarity assumption, the midplane values of all physical quantities scale as power laws in $\Psi$, and

$$
\Psi \propto r^{1 / \zeta}
$$

The magnetic flux distribution parameter $\zeta$ is denoted by $\xi$ in L96. The cold-wind model of BP82 has $\zeta=4 / 3$. In a disk that drives a magnetocentrifugal wind, $\zeta<4 / 3$, and if the outflow rate in the wind is much smaller than the accretion rate, as is the case in protostellar disks, then $\delta \zeta \equiv 4 / 3-\zeta \ll \zeta($ L96 $)$.

In terms of the self-similarity dimensionless variables $\varphi \equiv \Psi / \Psi_{*}$ and $s \equiv z / r$ (denoted by $t$ in L96), various physical quantities are normalized as follows:

$$
\begin{array}{lc}
r=r_{*} \varphi^{\zeta} x(s) ; & z=r_{*} \varphi^{\zeta} s x(s) ; \\
\rho=\rho_{*} \varphi^{2-3 \zeta} \tilde{\rho}(s) ; & \boldsymbol{V}=V_{\mathrm{K} *} \varphi^{-\frac{\zeta}{2}} \boldsymbol{v}(s) ; \\
\boldsymbol{B}=B_{z *} \varphi^{1-2 \zeta} \boldsymbol{b}(s) ; & \boldsymbol{E}=\frac{V_{\mathrm{K} *} B_{z *}}{c} \varphi^{1-\frac{5}{2} \zeta} \boldsymbol{e}(s) ; \\
\boldsymbol{E}_{\mathrm{c}}=\frac{V_{\mathrm{K} *} B_{z *}}{c} \varphi^{1-\frac{5}{2} \zeta} \boldsymbol{e}_{\mathrm{c}}(s) ; & P=P_{*} \varphi^{2-4 \zeta} p(s) ; \\
\boldsymbol{\sigma}=\frac{c^{2}}{4 \pi r_{*} V_{\mathrm{K} *}} \varphi^{-\frac{\zeta}{2}} \tilde{\boldsymbol{\sigma}}(s) ; & \boldsymbol{J}=\frac{c B_{z *}}{4 \pi r_{*}} \varphi^{1-3 \zeta} \boldsymbol{j}(s) .
\end{array}
$$

The variable $\varphi$ raised to various powers converts midplane values on the reference field line (subscript ' $*$ '), which is labeled by flux function value $\Psi_{*}$, to midplane values on a generic field line (subscript ' 0 ') with flux function value $\Psi=\Psi_{0}$. The combination $r_{*} \varphi^{\zeta}$, for example, is equal to $r_{0}$, the midplane cylindrical radius of the field line labeled by flux function value $\Psi_{0}$. The exponents for the power laws in $\varphi$ in Equation (21) come from dimensional analysis of the governing equations. The variable $s$ expresses position along a field line. The new coordinates $\{s, \varphi\}$ are expressed in terms of the spatial coordinates $\{r, z\}$ as $s=z / r$ and $\varphi=\left(\frac{1}{r_{*}} \frac{r}{x(z / r)}\right)^{1 / \zeta}$. This gives

$$
\begin{gathered}
\left(\frac{\partial s}{\partial r}\right)_{z}=-\frac{s}{r} ;\left(\frac{\partial s}{\partial z}\right)_{r}=\frac{1}{r} \\
\left(\frac{\partial \varphi}{\partial r}\right)_{z}=\frac{1}{\zeta} \frac{\varphi}{r}\left(\frac{x+x^{\prime} s}{x}\right) ;\left(\frac{\partial \varphi}{\partial z}\right)_{r}=\frac{1}{\zeta} \frac{\varphi}{r}\left(-\frac{x^{\prime}}{x}\right)
\end{gathered}
$$

In these relations, $x^{\prime} \equiv\left(\frac{\partial x}{\partial s}\right)_{\varphi}$. In terms of the new coordinates, the spatial derivatives of a generic quantity $A=A_{*} \varphi^{\alpha_{a}} a(s)$ take the following forms (Li 1995):

$$
\begin{aligned}
\left(\frac{\partial A}{\partial r}\right)_{z} & =\left(\frac{\partial A}{\partial s}\right)_{\varphi}\left(\frac{\partial s}{\partial r}\right)_{z}+\left(\frac{\partial A}{\partial \varphi}\right)_{s}\left(\frac{\partial \varphi}{\partial r}\right)_{z} \\
& =\frac{A_{*} \varphi^{\alpha_{a}}}{r_{*} \varphi^{\zeta}} \frac{\alpha_{a} a\left(x+x^{\prime} s\right)-\zeta a^{\prime} x s}{\zeta x^{2}}
\end{aligned}
$$

and

$$
\begin{aligned}
\left(\frac{\partial A}{\partial z}\right)_{r} & =\left(\frac{\partial A}{\partial s}\right)_{\varphi}\left(\frac{\partial s}{\partial z}\right)_{r}+\left(\frac{\partial A}{\partial \varphi}\right)_{s}\left(\frac{\partial \varphi}{\partial z}\right)_{r} \\
& =\frac{A_{*} \varphi^{\alpha_{a}}}{r_{*} \varphi^{\zeta}} \frac{\zeta a^{\prime} x-\alpha_{a} a x^{\prime}}{\zeta x^{2}}
\end{aligned}
$$

where $a^{\prime} \equiv\left(\frac{\partial a}{\partial s}\right)_{\varphi}$. 
The partial differential equations describing the disk can now be recast as ordinary differential equations and algebraic relations in the normalized quantities. The equation of mass continuity becomes

$$
\begin{aligned}
v_{z}^{\prime}-s v_{r}^{\prime}+\frac{v_{z}-v_{r} s}{\tilde{\rho}} \tilde{\rho}^{\prime} & =\left(\frac{5}{2}-\frac{2}{\zeta}\right) v_{r} \\
& +\left(\frac{2}{\zeta}-\frac{7}{2}\right) \frac{x^{\prime}}{x}\left(v_{z}-v_{r} s\right)
\end{aligned}
$$

and the conservation of momentum (Equation (8) ) becomes

$$
\begin{aligned}
\left(v_{z}-v_{r} s\right) & v_{r}^{\prime}-\frac{\theta s}{\tilde{\rho}} \tilde{\rho}^{\prime}=\frac{v_{r}^{2}}{2}+v_{\phi}^{2}-\left(v_{z}-v_{r} s\right) \frac{v_{r} x^{\prime}}{2 x} \\
& -\frac{1}{\left(1+s^{2}\right)^{3 / 2}}+\left(4-\frac{2}{\zeta}\right) \theta\left(1+\frac{x^{\prime} s}{x}\right) \\
& +\frac{\nu x}{\tilde{\rho}}\left(j_{\phi} b_{z}-j_{z} b_{\phi}\right)
\end{aligned}
$$

in the $r$ direction,

$$
\begin{aligned}
\left(v_{z}-v_{r} s\right) v_{\phi}^{\prime}= & -\frac{v_{r} v_{\phi}}{2}-\left(v_{z}-v_{r} s\right) \frac{v_{\phi} x^{\prime}}{2 x} \\
& +\frac{\nu x}{\tilde{\rho}}\left(j_{z} b_{r}-j_{r} b_{z}\right)
\end{aligned}
$$

in the $\phi$ direction, and

$$
\begin{aligned}
& \left(v_{z}-v_{r} s\right) v_{z}^{\prime}+\frac{\theta}{\tilde{\rho}} \tilde{\rho}^{\prime}=\frac{v_{r} v_{z}}{2}-\left(v_{z}-v_{r} s\right) \frac{v_{z} x^{\prime}}{2 x} \\
& -\frac{s}{\left(1+s^{2}\right)^{3 / 2}}-\left(4-\frac{2}{\zeta}\right) \theta \frac{x^{\prime}}{x}+\frac{\nu x}{\tilde{\rho}}\left(j_{r} b_{\phi}-j_{\phi} b_{r}\right)
\end{aligned}
$$

in the $z$ direction, where

$$
\theta \equiv c_{\mathrm{s}}^{2} / v_{\mathrm{K}}^{2}
$$

and

$$
\nu \equiv v_{\mathrm{A}, 0}^{2} / v_{\mathrm{K}, 0}^{2} .
$$

The quantity $v_{\mathrm{A}, 0}^{2} / v_{\mathrm{K}}^{2}$ is denoted by $\sigma$ in L96.

By definition, $\Psi=\Psi_{*} \varphi$. The relation $B_{r} r=-\left(\frac{\partial \Psi}{\partial z}\right)_{r}$ therefore gives $\left(B_{z *} \varphi^{1-2 \zeta} b_{r}\right)\left(r_{*} \varphi^{\zeta} x\right)=\frac{\Psi_{*} \varphi}{r_{*} \varphi^{\zeta}}\left(\frac{x^{\prime}}{\zeta x^{2}}\right)$. From the definition of the flux function, $\frac{\Psi_{*}}{r_{*}^{2}}=B_{z *}$, so we have

$$
x^{\prime}=b_{r} x^{3} .
$$

At a point $(r, z)=r_{*} \varphi^{\zeta}(x(s), s x(s))$ on a field line, the unit tangent vector along the field line is

$$
\frac{x^{\prime} \hat{r}+\left(x+x^{\prime} s\right) \hat{z}}{\sqrt{x^{\prime 2}+\left(x+x^{\prime} s\right)^{2}}}=\frac{b_{r} \hat{r}+b_{z} \hat{z}}{\sqrt{b_{r}^{2}+b_{z}^{2}}} .
$$

This relation, combined with Equation (31), gives

$$
b_{z}=b_{r} s+\frac{1}{x^{2}} \text {. }
$$

Equation (32), which expresses $b_{z}$ in terms of $b_{r}, x$, and $s$, automatically satisfies the solenoidal condition (Equation (11)).

Ampère's law (Equation (90) becomes

$$
b_{\phi}^{\prime}=\left(\frac{1}{\zeta}-2\right) \frac{x^{\prime}}{x} b_{\phi}-x j_{r}
$$

in the $r$ direction,

$$
b_{r}^{\prime}+s b_{z}^{\prime}=x j_{\phi}+\left(\frac{1}{\zeta}-2\right)\left[\frac{x^{\prime}}{x}\left(b_{r}+b_{z} s\right)+b_{z}\right]
$$

in the $\phi$ direction, and

$$
s b_{\phi}^{\prime}=b_{\phi}+\left(\frac{1}{\zeta}-2\right) b_{\phi}\left(1+\frac{x^{\prime} s}{x}\right)-x j_{z}
$$

in the $z$ direction. Using the algebraic relation for $b_{z}$, the $\phi$ component simplifies further to

$$
b_{r}^{\prime}=\frac{x j_{\phi}+b_{r} s+\left(\frac{1}{\zeta}-2\right)\left[\frac{x^{\prime}}{x}\left(b_{r}+b_{z} s\right)+b_{z}\right]}{1+s^{2}} .
$$

The poloidal components of Ampère's law (Equations (33) and (35) ) together imply

$$
j_{z}=j_{r} s+\left(\frac{1}{\zeta}-1\right) \frac{b_{\phi}}{x} .
$$

Ohm's law (Equation (1)) becomes

$$
\boldsymbol{j}=\tilde{\boldsymbol{\sigma}} \cdot \boldsymbol{e}_{\mathrm{c}}=\tilde{\sigma}_{\mathrm{O}} \boldsymbol{e}_{\mathrm{c} \|}+\tilde{\sigma}_{\mathrm{H}} \hat{b} \times \boldsymbol{e}_{\mathrm{c} \perp}+\tilde{\sigma}_{\mathrm{P}} \boldsymbol{e}_{\mathrm{c} \perp},
$$

where the comoving electric field is given by

$$
e_{\mathrm{c}}=\boldsymbol{e}+\boldsymbol{v} \times \boldsymbol{b}
$$

The three components of Equation (38) are

$$
j_{r}=\tilde{\sigma}_{\mathrm{O}} e_{\mathrm{c} r \|}+\frac{\tilde{\sigma}_{\mathrm{H}}}{b}\left(b_{\phi} e_{\mathrm{c} z \perp}-b_{z} e_{\mathrm{c} \phi \perp}\right)+\tilde{\sigma}_{\mathrm{P}} e_{\mathrm{c} r \perp}
$$

$$
j_{\phi}=\tilde{\sigma}_{\mathrm{O}} e_{\mathrm{c} \phi \|}+\frac{\tilde{\sigma}_{\mathrm{H}}}{b}\left(b_{z} e_{\mathrm{c} r \perp}-b_{r} e_{\mathrm{c} z \perp}\right)+\tilde{\sigma}_{\mathrm{P}} e_{\mathrm{c} \phi \perp},
$$

$$
j_{z}=\tilde{\sigma}_{\mathrm{O}} e_{\mathrm{c} z \|}+\frac{\tilde{\sigma}_{\mathrm{H}}}{b}\left(b_{r} e_{\mathrm{c} \phi \perp}-b_{\phi} e_{\mathrm{c} r \perp}\right)+\tilde{\sigma}_{\mathrm{P}} e_{\mathrm{c} z \perp},
$$

where $b=\sqrt{b_{r}^{2}+b_{\phi}^{2}+b_{z}^{2}}$, and the magnetic field-parallel and -perpendicular components of the comoving electric field satisfy

$$
\begin{gathered}
\frac{e_{\mathrm{c} r \|}}{b_{r}}=\frac{e_{\mathrm{c} \phi \|}}{b_{\phi}}=\frac{e_{\mathrm{c} z \|}}{b_{z}}, \\
e_{\mathrm{c} r \perp} b_{r}+e_{\mathrm{c} \phi \perp} b_{\phi}+e_{\mathrm{c} z \perp} b_{z}=0,
\end{gathered}
$$

and

$$
e_{\mathrm{c}}=e_{\mathrm{c} \|}+e_{\mathrm{c} \perp}
$$


The poloidal components of the induction equation (Equations (17) and (19)) yield the relation $e_{\phi}(r, z)=$ $e_{\phi}(r, 0)$, which implies

$$
e_{\phi}(s)=e_{\phi}(0) x(s)^{\frac{1}{\xi}-\frac{5}{2}} .
$$

The $\phi$ component of the induction equation (Equation (18) ) yields

$$
e_{r}^{\prime}+s e_{z}^{\prime}=\left(\frac{1}{\xi}-\frac{5}{2}\right)\left[\frac{x^{\prime}}{x}\left(e_{r}+e_{z} s\right)+e_{z}\right] .
$$

Combining equations (25), (26), and (28) yields

$$
\begin{aligned}
\tilde{\rho}^{\prime}= & {\left[\frac{\tilde{\rho}}{\left(v_{z}-v_{r} s\right)^{2}-\theta\left(1+s^{2}\right)}\right]\left[\left(2-\frac{2}{\zeta}\right) v_{r}\left(v_{z}-v_{r} s\right)\right.} \\
& +\left(\frac{2}{\zeta}-3\right) \frac{x^{\prime}}{x}\left(v_{z}-v_{r} s\right)^{2} \\
& +\left(4-\frac{2}{\zeta}\right) \theta\left(s+\frac{x^{\prime}}{x}+\frac{x^{\prime} s^{2}}{x}\right)+v_{\phi}^{2} s \\
& \left.+\frac{\nu x}{\tilde{\rho}}\left(j_{\phi}\left[b_{r}+b_{z} s\right]-b_{\phi}\left[j_{r}+j_{z} s\right]\right)\right] .
\end{aligned}
$$

This relation shows that the point where $\left(v_{z}-v_{r} s\right)^{2}=$ $\theta\left(1+s^{2}\right)$ is a singular point of the system of equations, since the denominator of $\tilde{\rho}^{\prime}$ vanishes there. The quantity $\sqrt{\left(v_{z}-v_{r} s\right)^{2} /\left(1+s^{2}\right)}$ is the component of the poloidal velocity perpendicular to the local surface of selfsimilarity (the surface of constant $s$ ), normalized by the Keplerian velocity. The denominator of $\tilde{\rho}^{\prime}$ vanishes when $\left(v_{z}-v_{r} s\right)^{2} /\left(1+s^{2}\right)=\theta=\left(c_{\mathrm{S}} / V_{\mathrm{K}}\right)^{2}$. This occurs when the unnormalized velocity component $V_{z}-V_{r} s / \sqrt{1+s^{2}}$ becomes equal to the local sound speed; this defines the sonic point. The Alfvén point is similarly a critical point of the cold ideal wind equations, and occurs where the same poloidal velocity component $V_{z}-V_{r} s / \sqrt{1+s^{2}}$ reaches the local Alfvén speed.

Combining Equation (48) with Equations (26) and (28) gives expressions for $v_{r}^{\prime}$ and $v_{z}^{\prime}$. Equation (131) gives $x^{\prime}$, Equation (27) gives $v_{\phi}^{\prime}$, and Equations (36) and (33) give $b_{r}^{\prime}$ and $b_{\phi}^{\prime}$, respectively. The final ordinary differential equation is Equation (47). Solving these 8 ordinary differential equations requires knowledge of the conductivity profile $\tilde{\sigma}_{\mathrm{O}}(s), \tilde{\sigma}_{\mathrm{H}}(s)$, and $\tilde{\sigma}_{\mathrm{P}}(s)$, which can be obtained using a known disk ionoization profile or some simplifying assumptions. In order to solve for $e_{r}^{\prime}$ in Equation (47), it is necessary to express $e_{z}$ as a function of the other variables and then differentiate the resulting relation. This expression is derived in Appendix A.

\subsubsection{Values at the Midplane}

All physical quantities are assumed to have either odd or even symmetry in $z$ about the midplane. The magnetic field is vertical at the midplane, so the $s$ derivative coincides with a $z$ derivative at that location.

By definition, $x_{0}=1$ and $\tilde{\rho}_{0}=1$. Since the magnetic field is vertical at the midplane, $b_{r, 0}=0$ and $b_{\phi, 0}=$ 0 , and since the vertical component of the flow velocity must be continuous across the midplane, $v_{z, 0}=0$. The midplane radial velocity and azimuthal electric field are given by

$$
\epsilon \equiv-V_{r, 0} / c_{\mathrm{s}}
$$

and

$$
\epsilon_{\mathrm{B}} \equiv-c E_{\phi, 0} / c_{\mathrm{S}} B_{z, 0} .
$$

In dimensionless form, these relations are $v_{r, 0}=-\epsilon \theta^{1 / 2}$ and $e_{\phi, 0}=-\epsilon_{\mathrm{B}} \theta^{1 / 2}$, which combines with Equation (46) to give

$$
e_{\phi}(s)=-\epsilon_{\mathrm{B}} \theta^{1 / 2} x(s)^{\frac{1}{\xi}-\frac{5}{2}} .
$$

The quantities $\epsilon$ and $\epsilon_{\mathrm{B}}$ are parameters of the model whose values are set by conditions that must be satisfied at special points in the flow (see Section 3). The definitions of $\epsilon$ and $\epsilon_{\mathrm{B}}$ given here are the same as in WK93; a factor of $\theta^{1 / 2}$ appears in the expressions for $v_{r, 0}$ and $e_{\phi, 0}$ because velocities here are normalized by $v_{\mathrm{K}}$ rather than by $c_{\mathrm{S}}$ as in WK93.

At the midplane, Equations (26) and (27) become

$$
\frac{v_{r, 0}^{2}}{2}+v_{\phi, 0}^{2}-1+\left(4-\frac{2}{\zeta}\right) \theta+\nu j_{\phi, 0}=0
$$

and

$$
\frac{v_{r, 0} v_{\phi, 0}}{2}+\nu j_{r, 0}=0
$$

respectively. Since the magnetic field is vertical at the midplane, $e_{\mathrm{c} r \|, 0}=0, e_{\mathrm{c} r \perp, 0}=e_{\mathrm{c} r, 0}=e_{r, 0}+v_{\phi, 0}$, $e_{\mathrm{c} \phi \|, O}=0$, and $e_{\mathrm{c} \phi \perp, 0}=e_{\mathrm{c} \phi, 0}=e_{\phi, 0}-v_{r, 0}=$ $\left(\epsilon-\epsilon_{\mathrm{B}}\right) \theta^{1 / 2}$. The radial and azimuthal midplane current densities are therefore $j_{r, 0}=-\tilde{\sigma}_{\mathrm{H}, 0} e_{\mathrm{c} \phi, O}+\tilde{\sigma}_{\mathrm{P}, 0} e_{\mathrm{c} r, O}$ and $j_{\phi, 0}=\tilde{\sigma}_{\mathrm{H}, 0} e_{\mathrm{c} r, o}+\tilde{\sigma}_{\mathrm{P}, 0} e_{\mathrm{c} \phi, o}$ (see Equations (40) and (41)). Substituting these relations into Equations (52) and (53) gives

$$
\begin{aligned}
& \frac{\epsilon^{2} \theta}{2}+v_{\phi, 0}^{2}-1+\left(4-\frac{2}{\zeta}\right) \theta \\
& +\nu\left(\tilde{\sigma}_{\mathrm{H}, 0}\left[e_{r, 0}+v_{\phi, 0}\right]+\tilde{\sigma}_{\mathrm{P}, 0}\left[\epsilon-\epsilon_{\mathrm{B}}\right] \theta^{\frac{1}{2}}\right)=0,
\end{aligned}
$$

and

$$
\begin{aligned}
& -\frac{\epsilon \theta^{\frac{1}{2}} v_{\phi, 0}}{2} \\
& +\nu\left(-\tilde{\sigma}_{\mathrm{H}, 0}\left[\epsilon-\epsilon_{\mathrm{B}}\right] \theta^{\frac{1}{2}}+\tilde{\sigma}_{\mathrm{P}, 0}\left[e_{r, 0}+v_{\phi, 0}\right]\right)=0 .
\end{aligned}
$$

Combining these two equations in the unknowns $v_{\phi, 0}$ and $e_{r, 0}$ gives

$$
\begin{aligned}
v_{\phi, 0}^{2}+v_{\phi, 0} & \left(\frac{\tilde{\sigma}_{\mathrm{H}, 0} \epsilon \theta^{\frac{1}{2}}}{2 \tilde{\sigma}_{\mathrm{P}, 0}}\right)+\left[\frac{\epsilon^{2} \theta}{2}-1+\left(4-\frac{2}{\zeta}\right) \theta\right. \\
& \left.+\nu\left(\frac{\tilde{\sigma}_{\mathrm{H}, 0}^{2}+\tilde{\sigma}_{\mathrm{P}, 0}^{2}}{\tilde{\sigma}_{\mathrm{P}, 0}}\right)\left(\epsilon-\epsilon_{\mathrm{B}}\right) \theta^{\frac{1}{2}}\right]=0,
\end{aligned}
$$

which can be solved for $v_{\phi, 0}$. Either Equation (54) or Equation (55) can then be used to calculate $e_{r, 0}$. 
TABLE 1

Summary of Midplane Values

\begin{tabular}{ccc}
\hline \hline Variable & Midplane Value & $s$-Derivative At Midplane \\
\hline$x$ & 1 & 0 \\
$\tilde{\rho}$ & 1 & 0 \\
$v_{r}$ & $-\epsilon \theta^{\frac{1}{2}}$ & 0 \\
$v_{\phi}$ & $v_{\phi, 0}$ & $-\left(\frac{5}{2}-\frac{2}{\xi}\right) \epsilon \theta^{\frac{1}{2}}$ \\
$v_{z}$ & 0 & $j_{\phi, 0}+\left(\frac{1}{\xi}-2\right)$ \\
$b_{r}$ & 0 & $-j_{r, 0}$ \\
$b_{\phi}$ & 0 & 0 \\
$b_{z}$ & 1 & 0 \\
$e_{r}$ & $\left(\frac{\epsilon \theta^{\frac{1}{2}}}{2 \nu \tilde{\sigma}_{\mathrm{P}, 0}}-1\right) v_{\phi, 0}+\frac{\tilde{\sigma}_{H, 0}}{\tilde{\sigma}_{P, 0}}\left(\epsilon-\epsilon_{\mathrm{B}}\right) \theta^{\frac{1}{2}}$ & 0 \\
$e_{\phi}$ & $-\epsilon_{\mathrm{B}} \theta^{\frac{1}{2}}$ & 0 \\
$j_{r}$ & $\frac{\epsilon \theta^{\frac{1}{2}} v_{\phi, 0}}{2 \nu}$ & 0 \\
$j_{\phi}$ & $\frac{1}{\nu}\left[1-\frac{\epsilon^{2} \theta}{2}-v_{\phi, 0}^{2}-\left(4-\frac{2}{\xi}\right) \theta\right]$ & \\
\hline
\end{tabular}

The values of several physical quantities and their $s$ derivatives at the midplane as functions of the model parameters and $v_{\phi, 0}$ are summarized in Table 1 .

Note that the expression for $v_{z, 0}^{\prime}$ is calculated using the mass continuity Equation (25), rather than Equation (28), which is identically 0 at the midplane. Also, from Equation (35) we have $j_{z, 0}=0$. The magnetic field is vertical at the midplane, so $e_{\mathrm{c} z, 0}=e_{\mathrm{c} z \|, 0}$. From Ohm's law (42) we have $j_{z, 0}=\tilde{\sigma}_{\mathrm{O}, 0} e_{\mathrm{c} z, 0}$; since $\tilde{\sigma}_{\mathrm{O}, 0}$ is non-zero, $j_{z, 0}=0$ implies $e_{\mathrm{c} z, 0}=0$.

The midplane is a singular point of the differential equations describing the disk, and special care is needed in initiating the numerical integration from that location. A common choice for dealing with this issue is to approximate the values of the dependent variables a small distance above the midplane using Taylor series expansions, a task that is made much easier by the assumption that all physical quantities have either even or odd symmetry about the midplane, which implies that the Taylor series for all quantities contain only even power terms or only odd power terms - for instance, the fact that $b_{r, 0}^{\prime} \neq 0$ immediately implies that $b_{r, 0}^{\prime \prime}=0$. Looking at Table 1 . it is clear that second-order Taylor series expansions for $v_{z}, b_{r}$ and $b_{\phi}$ only have one term apiece, corresponding to the first derivatives of those quantities at the midplane, which can be calculated using the expressions in Table 1 and Equation (56) for $v_{\phi, 0}$. The second-order Taylor series expansions for $x, \tilde{\rho}, v_{r}, v_{\phi}$, and $e_{r}$ include terms involving $x_{0}^{\prime \prime}, \tilde{\rho}_{0}^{\prime \prime}, v_{r, 0}^{\prime \prime}, v_{\phi, 0}^{\prime \prime}$, and $e_{r, 0}^{\prime \prime}$; the procedure for calculating these quantities is described in Appendix B.1.

\subsection{Ambipolar Diffusion Case}

The ambipolar conductivity regime occurs when all charged species $j$ have $\beta_{j} \gg 1$. Substituting into Equations (3)-(5) gives $\tilde{\sigma}_{O} \gg \tilde{\sigma}_{\mathrm{P}} \gg\left|\tilde{\sigma}_{\mathrm{H}}\right|$ in the ambipolar diffusion limit.

I assume that the charged species consist of ions (subscript ' $i$ ') and electrons (subscript 'e'). Charge neutrality and a comparison of the rate coefficients for electronneutral and ion-neutral momentum exchange given by Draine et al. (1993) imply that the heavier ions dominate the charge-neutral drag force. Combining this with the balancing of the charge-neutral drag and Lorentz forces yields

$$
\frac{\boldsymbol{J} \times \boldsymbol{B}}{c \rho}=\gamma \rho_{\mathrm{i}}\left(\boldsymbol{V}_{\mathrm{i}}-\boldsymbol{V}\right),
$$

where $\boldsymbol{V}_{\mathrm{i}}$ is the ion velocity and $\rho_{\mathrm{i}}$ is the ion mass density.

For a disk in the ambipolar diffusion limit where the only charged species are ions and electrons, the Elsasser number is $\Lambda=\gamma \rho_{\mathrm{i}} / \Omega_{\mathrm{K}} \equiv \Upsilon$, the ratio of the dynamic timescale to the neutral-ion momentum exchange time (Königl \& Salmeron 2011). This ratio is denoted by $\eta$ in L96. The neutral-field coupling parameter $\Upsilon$ in this case is related to the Pedersen conductivity by $\Upsilon=\left(4 \pi v_{\mathrm{A}}^{2} / c^{2}\right)\left(\sigma_{\mathrm{P}} / \Omega_{\mathrm{K}}\right)$ (SKW07).

The tight coupling of the ions to the magnetic field in the ambipolar diffusion limit implies that the comoving electric field vanishes in the frame of the ions. The electric field in the disk is therefore

$$
\boldsymbol{E}=-\frac{1}{c} \boldsymbol{V}_{\mathrm{i}} \times \boldsymbol{B} .
$$

\subsubsection{Governing Equations}

Equations (12) and (58) yield the induction equation

$$
\frac{\partial \boldsymbol{B}}{\partial t}=\nabla \times\left(\boldsymbol{V}_{\mathrm{i}} \times \boldsymbol{B}\right) .
$$

From Equation (58) and the definition of $\epsilon_{\mathrm{B}}, c E_{\phi}=$ $V_{\mathrm{i} r} B_{z}-V_{\mathrm{i} z} B_{r}=-\epsilon_{\mathrm{B}} c_{\mathrm{s}}(r) B_{z}(r, z=0)$, or

$$
V_{\mathrm{i} z} B_{r}=V_{\mathrm{i} z} B_{z}+\epsilon_{\mathrm{B}} c_{\mathrm{s}} B_{z}(r, z=0),
$$

where all quantities [except the midplane quantity $\left.B_{z}(r, z=0)\right]$ are evaluated at a point $(r, z)$ on the field line of interest.

The $\phi$ component of the induction Equation (12) is

$$
c\left(\frac{\partial E_{z}}{\partial r}-\frac{\partial E_{r}}{\partial z}\right)=0
$$

or

$$
\frac{\partial}{\partial r}\left(V_{\mathrm{i} \phi} B_{r}-V_{\mathrm{i} r} B_{\phi}\right)=\frac{\partial}{\partial z}\left(V_{\mathrm{i} z} B_{\phi}-V_{\mathrm{i} \phi} B_{z}\right) .
$$




\subsubsection{Self-Similarity Equations}

The ordinary differential equations obtained under the radial self-similarity assumption are identical to Equations (12)-(14) and (16)-(21) in L96. The equations used here differ from those in L96 only in the treatment of the induction equation; I allow for a nonzero $\epsilon_{\mathrm{B}}$ (or, equivalently, a nonzero $E_{\phi}$ ), which changes the usual flux-freezing condition for the ions. As a result, Equation (10) in L96 is replaced by Equations (60) and (62). Equations (22)-(24) in L96 (the self-similar, nondimensionalized versions of Equation (10) in L96) are replaced by the self-similar, nondimensionalized versions of Equation (60),

$$
v_{\mathrm{i} z} b_{r}=v_{\mathrm{i} r} b_{z}+\epsilon_{\mathrm{B}} \theta^{1 / 2} x^{1 / \zeta-5 / 2},
$$

and Equation (62),

$$
\begin{gathered}
v_{\mathrm{i} r}^{\prime} b_{\phi}-v_{\mathrm{i} \phi}^{\prime} b_{r} \\
-b_{r}^{\prime} \frac{b_{\phi}}{b_{r}}\left(v_{\mathrm{i} r}+\epsilon_{\mathrm{B}} \theta^{1 / 2} x^{1 / \zeta-1 / 2}\right) \\
+b_{\phi}^{\prime}\left(v_{\mathrm{i} r}+\epsilon_{\mathrm{B}} \theta^{1 / 2} x^{1 / \zeta-1 / 2}\right)= \\
b_{r} x^{2}\left(v_{\mathrm{i} r} b_{\phi}-v_{\mathrm{i} \phi} b_{r}\right),
\end{gathered}
$$

where the normalization scheme from Section 2.1.2 is used, with the addition of

$$
\boldsymbol{V}_{\mathrm{i}}=v_{\mathrm{K} *} \varphi^{-\frac{\zeta}{2}} \boldsymbol{v}_{\mathrm{i}}(s) .
$$

Taking the dot product of $\boldsymbol{B}$ with both sides of Equation (57) gives $\boldsymbol{B} \cdot\left(\boldsymbol{V}_{\mathrm{i}}-\boldsymbol{V}\right)=0$, which results in the following algebraic relation for $v_{\mathrm{i} \phi}$ :

$$
\begin{aligned}
v_{\mathrm{i} \phi} b_{\phi}= & b_{r} v_{r}+b_{\phi} v_{\phi}+b_{z} v_{z}-v_{\mathrm{i} r}\left(b_{r}+\frac{b_{z}^{2}}{b_{r}}\right) \\
& -\epsilon_{\mathrm{B}} \theta^{1 / 2} \frac{b_{z}}{b_{r}} x^{1 / \zeta-5 / 2},
\end{aligned}
$$

which can be differentiated to give $v_{\mathrm{i} \phi}^{\prime}$ for use in Equation (64).

I now have a system of 8 ordinary differential equations in the variables $x, \tilde{\rho}, v_{r}, v_{\phi}, v_{z}, v_{\mathrm{i} r}, b_{r}$ and $b_{\phi}$. This system differs from that of L96 in the treatment of the induction equation. A solution of this version of the disk equations and a matching wind solution are presented in Section 4. That solution assumes a functional form for the matter-field coupling parameter $\Upsilon \propto \tilde{\rho}_{\text {i }}$ that differs from the one used in L96-while L96 assumes that $\Upsilon$ increases monotonically with height, I take $\Upsilon$ to be constant along a field line, as would be the case when the ion density is constant (WK93). In fact, the coupling parameter is expected to decrease with height in the upper reaches of real protostellar disks not too far from the origin (Salmeron \& Wardle 2005), reflecting the decrease of the ion mass density $\tilde{\rho}_{\mathrm{i}}$ with height (which occurs even though the ionization fraction increases with height because the neutral mass density $\tilde{\rho}$ drops rapidly with $z$ ).

On the other hand, the coupling parameter increases with radial position because the Keplerian frequency $\Omega_{\mathrm{K}}$ decreases. Taking $\Upsilon$ constant is a compromise between these competing effects as the integration proceeds upwards and outwards along a field line. Finally, the solution presented here differs from the solutions presented in L96 in that I initiate the numerical integration of the disk equations using a Taylor series expansion (see Appendix B.2), whereas L96 uses the approximation $v_{z}=v_{r} s$ in a small region near the midplane to obtain algebraic relations for $v_{r}$ and $v_{\phi}$.

\subsubsection{Values at the Midplane}

From Equation (63), $v_{\mathrm{i} r, 0}=-\epsilon_{\mathrm{B}} \theta^{1 / 2}$. The $r$ component of momentum conservation (Equation (8)), with Equation (57) used to replace the Lorentz force term with an ion-neutral drag force term, yields Equation (13) of L96. At the midplane, this equation reduces to

$$
\frac{v_{r, 0}^{2}}{2}+v_{\phi, 0}^{2}-1+\left(4-\frac{2}{\zeta}\right) \theta+\Upsilon_{0}\left(v_{\mathrm{i} r, 0}-v_{r, 0}\right)=0
$$

or

$$
v_{\phi, 0}^{2}=1-\frac{\epsilon^{2} \theta}{2}-\left(4-\frac{2}{\zeta}\right) \theta+\Upsilon_{0} \theta^{\frac{1}{2}}\left(\epsilon-\epsilon_{\mathrm{B}}\right),
$$

which gives $v_{\phi, 0}$ in terms of the parameters $\theta, \zeta, \Upsilon_{0}$, $\epsilon$, and $\epsilon_{\mathrm{B}}$. Continuity of $v_{\mathrm{i} z}$ implies $v_{\mathrm{i} z, 0}=0$, and the $\phi$ component of the momentum equation (cf. Equation (14) of L96) evaluated at the midplane gives

$$
\Upsilon_{0}\left(v_{\mathrm{i} \phi, 0}-v_{\phi, 0}\right)=\frac{v_{r, 0} v_{\phi, 0}}{2},
$$

which can be used to calculate $v_{\mathrm{i} \phi, 0}$.

Combining Ampère's law (Equation (9) ) with Equation (57) yields equations for $b_{r}^{\prime}$ and $b_{\phi}^{\prime}$ (cf. Equations (17)(19) of L96); evaluating at the midplane yields

$$
b_{r, 0}^{\prime}=\frac{\Upsilon_{0}}{\nu}\left(v_{\mathrm{i} r, 0}-v_{r, 0}\right)-\left(2-\frac{1}{\zeta}\right)
$$

and

$$
b_{\phi, 0}^{\prime}=\frac{\Upsilon_{0}}{\nu}\left(v_{\mathrm{i} \phi, 0}-v_{\phi, 0}\right) .
$$

The expression for $v_{z, 0}^{\prime}$ (see Table1) remains unchanged.

The Taylor expansion procedure used to initiate the integration is described in Appendix B.2.

\section{MODEL PARAMETER CONSTRAINTS}

\subsection{Model Parameters}

The dimensionless parameters $\theta \equiv c_{\mathrm{S}}^{2} / v_{\mathrm{K}}^{2}$ and $\nu \equiv$ $v_{\mathrm{A}, 0}^{2} / v_{\mathrm{K}}^{2}$ are model inputs, as are the conductivity tensor component profiles $\sigma_{\mathrm{O}}(s), \sigma_{\mathrm{H}}(s)$, and $\sigma_{\mathrm{P}}(s)$. Alternatively, values for $\theta$ and $\nu$ together with a profile $\Upsilon(s)$ for the neutral-field coupling parameter suffices for the case of a disk in the ambipolar diffusion regime. In either case, a disk/wind solution involves constraining three parameters to satisfy certain conditions imposed by physical processes outside the disk. The first constrained parameter is $\zeta$, the power-law index of the magnetic flux distribution (Equation (20) ). The second is $\epsilon$, the normalized midplane value of $V_{r}$ (Equation (49)). The third is $\epsilon_{\mathrm{B}}$, the normalized midplane value of $E_{\phi}$ (Equation (50) ).

Two of the conditions to be satisfied are that the disk solution pass smoothly through the sonic point and match onto a wind solution that passes smoothly through 
the Alfvén point. The third condition relates the angle of the magnetic field at the disk surface to the distribution of the magnetic field exterior to the disk (see Section 3.3).

\subsection{Matching to a Wind Solution}

The sonic point $\left(z=z_{\mathrm{sp}}\right)$ is a singular point of the system of equations describing the disk (see Equation (48) and subsequent discussion), so it is not possible to integrate directly into the sonic point, but it is possible to get close. I run a bisection routine on $\epsilon$, holding $\zeta$ and $\epsilon_{\mathrm{B}}$ fixed, until I obtain a disk solution that accelerates smoothly almost all the way up to the sonic point. I then extrapolate the solution to $z_{\mathrm{sp}}$ and attempt to match the disk solution to a BP82-type wind solution, using the procedure outlined in SKW11. That is, I calculate the value of the wind parameter $\kappa$ (the normalized mass-tomagnetic flux ratio) using the disk solution at the sonic point, and I calculate $B_{r, \mathrm{~s}} / B_{z, \mathrm{~s}}$ and $\lambda_{\mathrm{s}}$, respectively the ratio of the radial and vertical components of the magnetic field ( $\xi_{\mathrm{b}}^{\prime}$ in the notation of SKW11) and the total specific angular momentum, using the disk solution at the disk surface. Here, the disk surface is taken to be the point where the azimuthal neutral gas velocity matches the local Keplerian rotation rate. I run a bisection routine on $\zeta$ until the values of $\kappa, \lambda$ and $\xi_{\mathrm{b}}^{\prime}$ calculated from the disk solution match the values at the base of a wind solution.

The matching procedure used here guarantees continuity of the magnetic field components, the poloidal mass flux, and the total specific angular momentum. However, it should be pointed out that the electric field is, in general, not continuous at the transition from the disk solution to the wind solution: in particular, there is a non-zero azimuthal comoving electric field $E_{\mathrm{c} \phi}$ in the disk solution because the parameter $\epsilon_{\mathrm{B}}$ is generally non-zero, but the cold, ideal MHD equations describing the wind solution have $E_{\mathrm{c} \phi}=0$ identically. In addition, the BP82 wind solution is derived for a razor-thin disk that has vanishing radial and vertical gas velocities at the base of the wind (negligible compared with the azimuthal velocity, which is exactly Keplerian at the base of the wind); these are good approximations when the region of interest is the large-scale outflow, but break down on small scales comparable to the thickness of the disk. The wind solution is therefore expected to give physically uninteresting results very close to the disk surface. In fact, the wind solution has a singular point at the midplane just as the disk solution does. I follow BP82 in integrating the wind equations downwards starting from the Alfvén point and stopping very close to the disk surface, where the solution is matched to a Taylor expansion. I then interpolate between the disk solution at the top of the disk and the wind solution at a distance $\chi / \chi_{\mathrm{A}}=0.002$ above the disk surface, where $\chi \equiv z / r_{\mathrm{s}}, r_{\mathrm{s}}$ is the radial distance of the disk surface along the field line of interest, and $\chi_{A}$ is the value of $\chi$ at the Alfvén point.

The wind solution extends past the Alfvén point. There is a second critical point of the equations for a selfsimilar wind corresponding to the modified fast magnetosonic point (BP82). However, before a cold BP82-type wind solution reaches the modified fast magnetosonic point, the flow "over-collimates" as the field lines bend back towards the axis of symmetry. This unphysical behavior results from ignoring heating of the jet ma- terial and thermal pressure support in the cold wind model. "Warm" self-similar jet models whose solutions do cross the modified fast magnetosonic have been found by Vlahakis et al. (2000) and Ferreira \& Casse (2004). For this study, I terminate the wind solution when the field lines begin to bend back towards the jet axis.

\subsection{The Disk Surface Field Constraint}

OL01 pointed out that in a self-consistent, global model of a magnetized disk, the inclination of the poloidal field at the disk surface can be determined under the approximation that the external magnetic field is nearly potential $(\nabla \times \boldsymbol{B} \approx 0)$. An analytic expression relating $B_{r, \mathrm{~s}}$ to the distribution of $B_{z, \mathrm{~s}}$ along the disk surface was derived, under this approximation and assuming an infinitely thin disk, by Ciolek \& Mouschovias (1993), who modeled magnetically supported, self-gravitating molecular cloud cores, and by Lubow et al. (1994a), who modeled flux advection in viscous accretion disks. The poloidal field inclination at the disk's surface is directly related to the balance between the inward advection of magnetic flux by the accretion flow and the outward diffusion of magnetic flux through the non-ideal disk. This connection follows from the fact that the radial component of the magnetic force (per unit area) at the disk surface, which is $\propto B_{r, \mathrm{~s}} B_{z, \mathrm{~s}}$, is balanced in the ambipolar diffusion regime by the radial ion-neutral drag force, which is $\propto V_{\mathrm{i} r}-V_{r}$ (or, taking the midplane values, $\propto \epsilon-\epsilon_{\mathrm{B}}$, which indicates the explicit relation of $b_{r, \mathrm{~s}}$ to the disk model parameters). Determining $B_{r, \mathrm{~s}}$ selfconsistently in this manner generally implies a nonzero value of $\epsilon_{\mathrm{B}}$. The relevant expression for $B_{r, \mathrm{~s}}$ is

$$
\begin{aligned}
& r B_{r, \mathrm{~s}}(r)= \\
& \int_{0}^{\infty} d k k J_{1}(k r) \int_{0}^{\infty} d r^{\prime} r^{\prime}\left[B_{z, 0}\left(r^{\prime}\right)-B_{\mathrm{ref}}\right] J_{0}\left(k r^{\prime}\right),
\end{aligned}
$$

where $J_{0}$ and $J_{1}$ are Bessel functions of the first kind of order 0 and 1 , respectively, $B_{z, 0}(r)$ is the midplane vertical field component at radius $r$, which is assumed to remain constant up to the surface [so that $B_{z, \mathrm{~s}}(r)=$ $\left.B_{z, 0}(r)\right]$, and $B_{\text {ref }}$ is the external magnetic field at "infinity," which is taken to be $\ll B_{z, 0}(r)$, so it may be safely ignored in the second integral.

For a power-law profile $B_{z, 0} \propto r^{-a}$, Equation (71) yields $B_{r, \mathrm{~s}}(r) / B_{z, \mathrm{~s}}(r)=[\Gamma(1-a / 2) \Gamma(1 / 2+$ $a / 2)][\Gamma(a / 2) \Gamma(3 / 2-a / 2)]$ for $\operatorname{Re}\{a\} \in(-3 / 2,-1 / 2)$, where $\Gamma$ is the gamma function. The power-law exponent is related to the disk model parameter $\zeta$ by $a=2-1 / \zeta$, so

$$
\frac{B_{r, \mathrm{~s}}(r)}{B_{z, \mathrm{~s}}(r)}=\frac{\Gamma[1 /(2 \zeta)] \Gamma[3 / 2-1 /(2 \zeta)]}{\Gamma[1-1 /(2 \zeta)] \Gamma[1 / 2+1 /(2 \zeta)]} .
$$

This result holds for $\zeta \in(2 / 3,2)$ and is therefore appropriate for the values $\zeta \lesssim 4 / 3$ that are expected to characterize physically viable solutions (e.g., L96; F97). For $\zeta=4 / 3$, Equation (72) yields $B_{r, \mathrm{~s}}(r) / B_{z, \mathrm{~s}}(r)=1.428$.

Once a disk solution that passes smoothly through the sonic point has been matched to a wind solution, I bisect on $\epsilon_{\mathrm{B}}$ until the value of $B_{r, \mathrm{~s}}$ matches the value imposed by the field distribution.

\section{ILLUSTRATIVE SOLUTION}




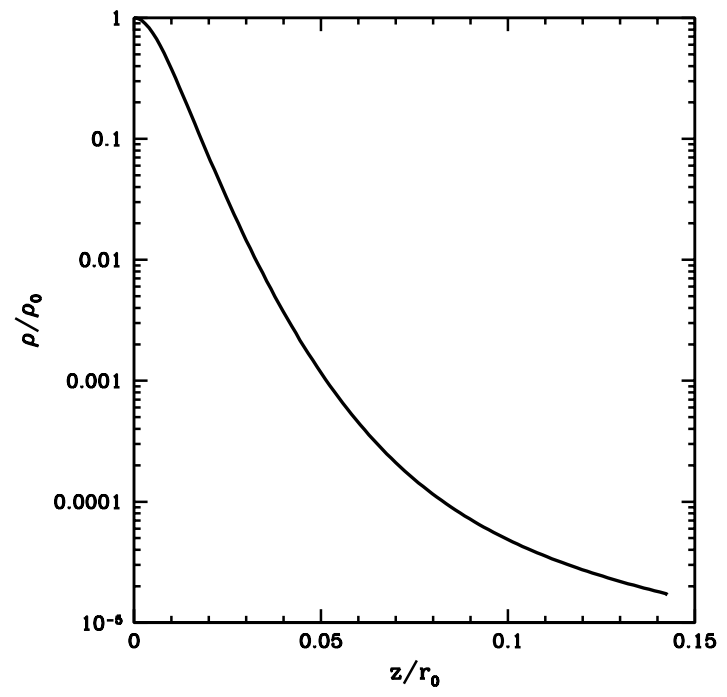

FIG. 1.- Density structure of the constant $\Upsilon$ disk solution.

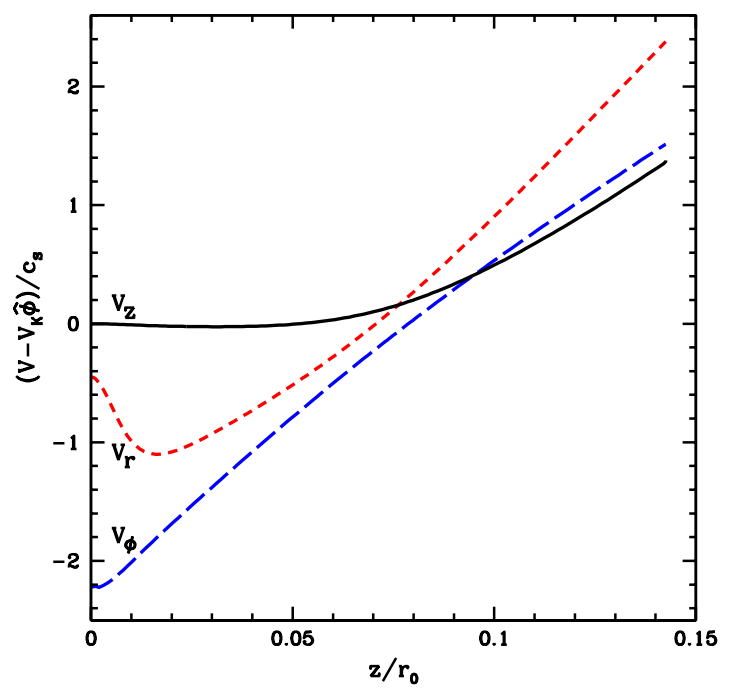

FIG. 2.- Neutral velocity structure of the constant $\Upsilon$ disk solution. The Keplerian velocity has been subtracted from the azimuthal component, and the plotted velocities are normalized by the local sound speed.

Protostellar disks have typical inferred temperatures at $1 \mathrm{AU}$ of $\sim 100-300 \mathrm{~K}$, masses of $\sim 0.005-0.2 M_{\odot}$, column densities at $5 \mathrm{AU}$ of $\sim 0.3-300 \mathrm{~g} \mathrm{~cm}^{-2}$, and extend to radii $\sim 200 \mathrm{AU}$ (e.g., Andrews \& Williams 2007). Disk/wind model solutions typically have $v_{\mathrm{A}, 0} / c_{\mathrm{S}} \lesssim 1$ (e.g., WK93), and observations of protostellar systems with jets indicate that the ratio of the outflow and accretion rates is $\sim 0.01-0.1$ (e.g., Hartigan et al. 1995). Finally, observations of remanent magnetization of meteorite samples suggest a magnetic field of $\sim 1 \mathrm{G}$ at $3 \mathrm{AU}$ in the early solar nebula (Levy \& Sonett 1978). Motivated by these facts, I choose parameter values $\theta=1 \times 10^{-3}$ and $\nu=7.744 \times 10^{-4}$. These values together with the constant coupling profile $\Upsilon(s)=10$ yield a solution of the disk equations described in Section 2.2. This solution passes smoothly through the sonic point, matches to a

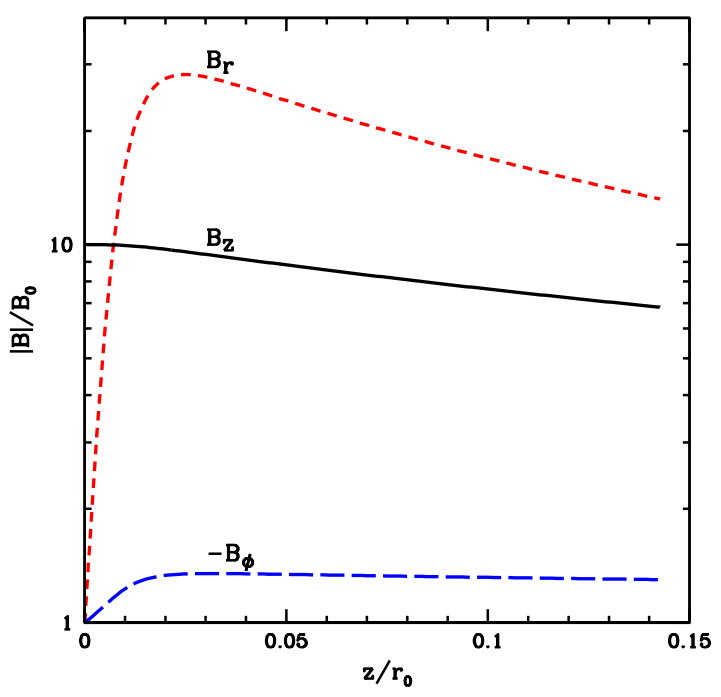

FIG. 3.- Magnetic field structure of the constant $\Upsilon$ disk solution. All components are normalized by the midplane field strength.

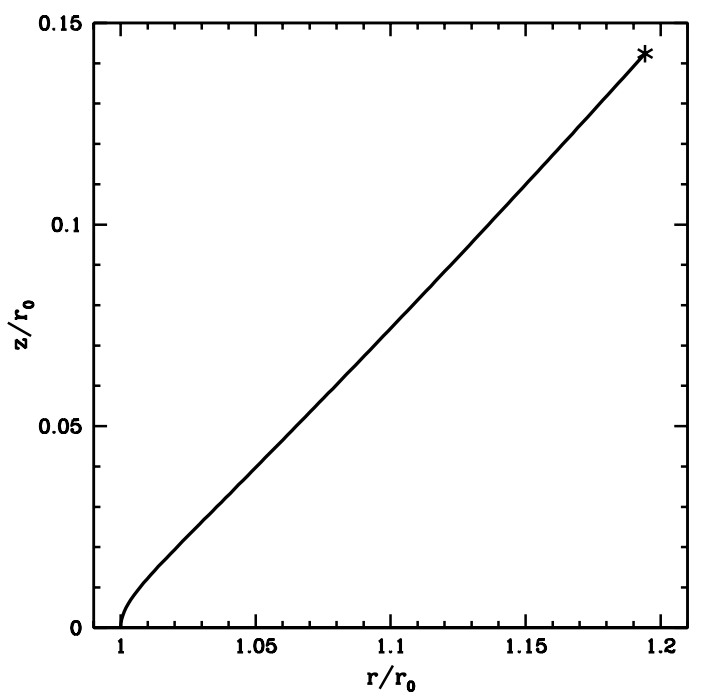

Fig. 4.- Poloidal magnetic field line shape of the constant $\Upsilon$ disk solution. The location of the sonic point is marked ('*').

wind solution that passes smoothly through the Alfvén point, and satisfies the constraint on the magnetic field at the surface of the disk. The corresponding disk and wind parameters are $\delta \zeta=3.05 \times 10^{-3}, \epsilon=0.45, \epsilon_{\mathrm{B}}=0.03$, $\kappa=1.27 \times 10^{-3}, \lambda=108.4$, and $\xi_{\mathrm{b}}^{\prime}=1.42$. The small value of $\delta \zeta$ found here is consistent with the predictions of L96 and F97 that cold wind-driving disks should have field-line distributions very close to the limiting case of $\zeta=4 / 3$ (but see Casse \& Ferreira 2000 for arguments that larger values of $\delta \zeta$ can occur in wind-driving disks when thermal effects are considered $\left.{ }^{3}\right)$.

3 The quantity $\delta \zeta$ is related to the quantity $\xi$ in Casse \& Ferreira $(2000)$ as $\delta \zeta \approx 8 / 9 \xi$; the ratio $f$ of the mass outflow in the wind between radii $r_{\text {in }}$ and $r_{\max }$ to the mass accretion rate at $r_{\text {in }}$ is $f=\left(r_{\max } / r_{\text {in }}\right)^{\xi}-1$. For $\left(r_{\max } / r_{\text {in }}\right) \approx 1000$ and an observed $f \sim 0.01-0.1, \xi$ is inferred to lie in the range $\xi \sim 0.0014-0.014$. Of the two representative solutions in Casse \& Ferreira (2000), the one with almost no entropy generation gives $\xi \sim 0.001$, while the 


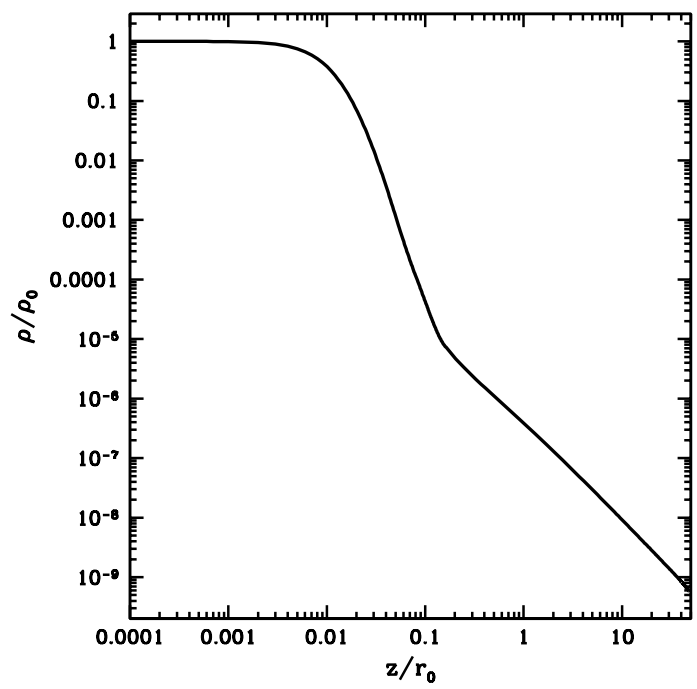

FIG. 5.- Density structure of the constant $\Upsilon$ disk solution and the matched wind solution.

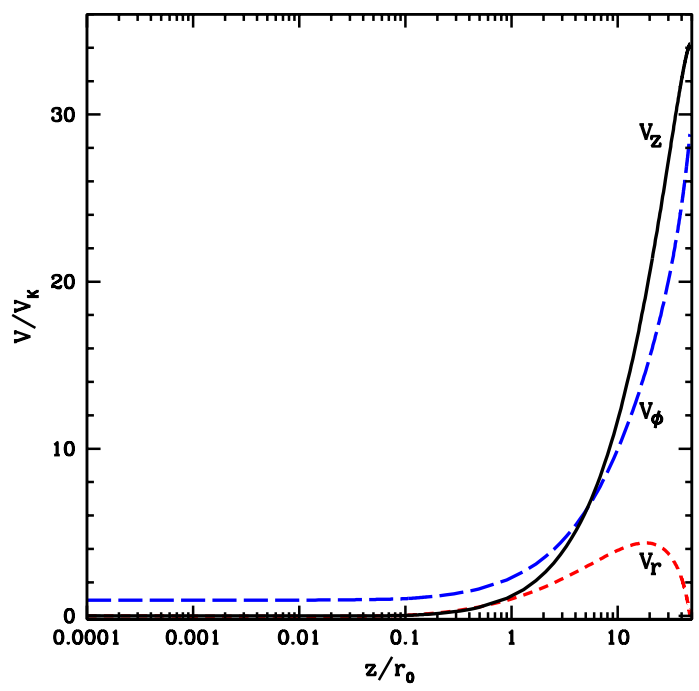

FIG. 6.- Velocity structure of the constant $\Upsilon$ disk solution and the matched wind solution. The plotted velocities are normalized by the local Keplerian velocity.

A rough estimate of the ratio of the mass outflow rate from both sides of the disk, between an outermost radius $r_{\max }$ and a given radius $r$, to the mass accretion rate through the disk at $r$, can be derived from Equation (89) of SKW11 as $\dot{M}_{\text {wind }} / \dot{M}_{\text {disk }} \approx \ln \left(r_{\max } / r\right) /[2(\lambda-1)]$; for the derived solution, and taking $r_{\max } / r \approx 1000$, this ratio is $\approx 3.2 \times 10^{-2}$. For a central protostar of mass $0.5 M_{\odot}$, the model has a column density of $\approx 42 \mathrm{~g} \mathrm{~cm}^{-2}$ at 1 AU and a column density of $\approx 19 \mathrm{~g} \mathrm{~cm}^{-2}$ at $5 \mathrm{AU}$ if the density is normalized so that the accretion rate is $\approx 3 \times 10^{-6} M_{\odot} \mathrm{yr}^{-1}$ and the total disk mass out to 200 $\mathrm{AU}$ is $\approx 0.055 M_{\odot}$. In comparison, the minimum mass solar nebula model (Hayashi 1981) has a column density

one with strong entropy generation gives $\xi \sim 0.5$, in which case almost all of the disk mass flows out into the wind rather than accreting onto the central protostar.

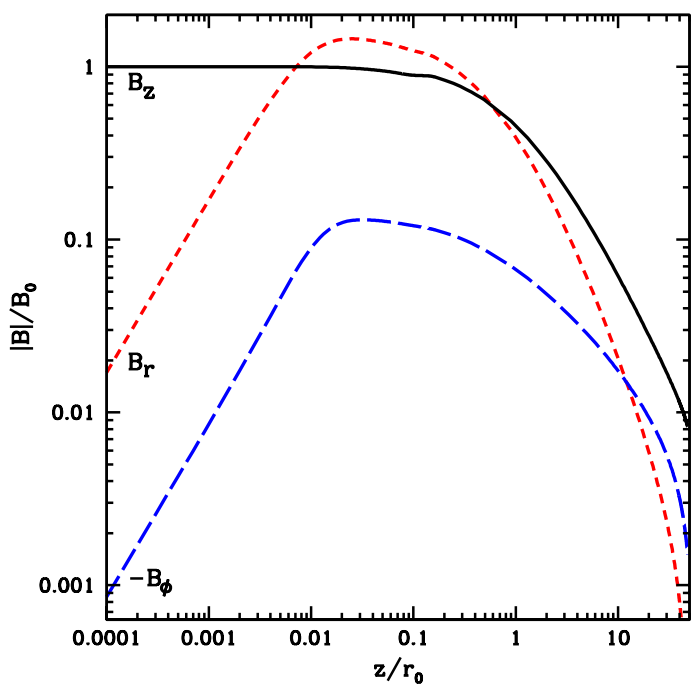

FIG. 7.- Magnetic field structure of the constant $\Upsilon$ disk solution and the matched wind solution. All components are normalized by the midplane field strength. The radial and toroidal components are 0 at the midplane, and the vertical component is 1 at the midplane, but all three components change rapidly within the disk, which extends up to $z / r_{0} \approx 0.079$

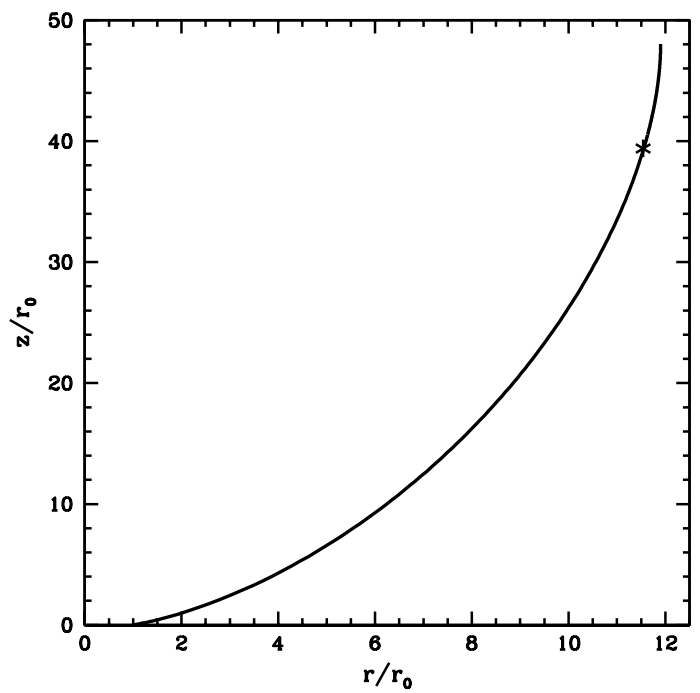

FIG. 8. - Poloidal magnetic field line shape of the constant $\Upsilon$ disk solution and the matched wind solution. The location of the Alfvén point is marked ('*').

at $1 \mathrm{AU}$ of $\approx 1700 \mathrm{~g} \mathrm{~cm}^{-2}$ and at $5 \mathrm{AU}$ of $\approx 150 \mathrm{~g} \mathrm{~cm}^{-2}$. The density normalization used here corresponds to a midplane magnetic field strength at $1 \mathrm{AU}$ of $|\boldsymbol{B}| \approx 2.5 \mathrm{G}$ and at $3 \mathrm{AU}$ of $\approx 0.6 \mathrm{G}$. The disk temperature at $1 \mathrm{AU}$ is $\approx 125 \mathrm{~K}$.

The gas density, velocity, and magnetic field structures of the disk solution as functions of position along a field line are shown in Figures 1 13 . The plots extend from the midplane, $z=0$, to the sonic point, $z_{\mathrm{sp}} \approx 0.14 r_{0}\left(\approx 3.8 h_{\mathrm{T}}\right)$. The location of the sonic point is marked in Figure 4, which shows the poloidal magnetic field line structure in the disk. The magnetic field components and the density are normalized by the midplane values of $B$ and $\rho$, respectively. The velocity compo- 
nents, with the Keplerian velocity subtracted from the azimuthal neutral and ion velocities, are normalized by the local value of the sound speed along the field line (that is, taking into account the spatial variation of the sound speed).

At the midplane, the field is vertical by symmetry; above the midplane, the radial component grows rapidly, a small negative toroidal component develops, and the vertical component decreases slightly. The vertical velocity $V_{z}$ starts at zero at the midplane, decreases to small negative values in the near-midplane region (reflecting the radial convergence of the accretion flow), and then increases back through zero at $z \approx 0.054 r_{0}\left(\approx 1.5 h_{\mathrm{T}}\right)$, becoming comparable to $c_{\mathrm{s}}$ at $z \approx 0.112 r_{0}\left(\approx 3.1 h_{\mathrm{T}}\right)$. The radial velocity starts out at $-0.45 c_{\mathrm{s}}$ at the midplane and decreases in the near-midplane region, reaching a minimum at $z \approx 0.016 r_{0}\left(\approx 0.5 h_{\mathrm{T}}\right)$ and then increasing, switching from an inflow to an outflow at $z \approx 0.071 r_{0}\left(\approx 2 h_{\mathrm{T}}\right)$. The azimuthal velocity is subKeplerian at the midplane $\left(\approx 0.93 V_{\mathrm{K}, 0}\right)$ but increases smoothly with height, becoming super-Keplerian at the disk surface, $z_{\mathrm{s}} \approx 0.77 r_{0}\left(\approx 2.2 h_{\mathrm{T}}\right)$, slightly above the point where $V_{r}$ becomes positive. In the hydrostatic approximation $\left(V_{z}=0\right), V_{r}=0$ and $V_{\phi}=V_{\mathrm{K}}$ at the same point (WK93). The effective density scale height $h_{\text {eff }}$ (defined so that $\left.\rho\left(z=h_{\text {eff }}\right) / \rho(z=0)=1 / \sqrt{e}\right)$ is $h_{\text {eff }} \approx 0.22 h_{\mathrm{T}}$, indicating that magnetic squeezing has a significant effect on the disk structure (WK93).

The gas density, velocity, and magnetic field structures of the matched disk and wind solutions as functions of position along a field line are shown in Figures [5 [7] The plots extend from $z=0$ to $z \approx 48 r_{0}$, where the field lines begin to bend back towards the jet axis and the wind solution terminates. Along the way, the wind solution passes through the Alfvén point, located at $z \approx 38.9 r_{0}$. Figures 57 are shown with the variable $z / r_{0}$ plotted on a logarithmic axis so that the structures of the disk and wind regions can be distinguished. The location of the Alfvén point is marked in Figure 8, which shows the poloidal magnetic field line structure in the disk and wind. In the region above the disk, the poloidal field quickly drops while the azimuthal field amplitude first grows slightly ( $B_{\phi}$ becomes more negative) and then decreases, in accordance with the expected behavior of a wind-driving disk. The vertical and azimuthal velocities increase smoothly throughout the outflow, while the radial velocity reaches a maximum and begins decreasing well below the Alfvén point. Note that Figure 6] differs from Figure 2 in that the unmodified azimuthal velocity is plotted and the velocities are normalized by the local value of the Keplerian speed (that is, taking into account the spatial variation of the Keplerian speed).

The solution presented here is similar to the WK93 model in that it considers a strongly coupled, ambipolar diffusive disk, but differs in that it is a global model with a non-zero value of $\epsilon_{\mathrm{B}}$ determined self-consistently from a constraint on the field line inclination at the disk surface. The equilibrium solution curves for the present model are undoubtedly modified from those of K04, but on the assumption that the changes are not drastic, a comparison of the representative solution and the results given in K04 may be instructive. The representative solution given here has $\kappa=1.27 \times 10^{-3}, \lambda=108.4$, and $a \equiv(\sigma / \theta)^{1 / 2}=0.88$; the quantity $\eta$ in K04 corresponds to $\Upsilon$. Comparing these values to Figures 1 and 2 of K04, it appears that the representative solution given here lies near the lowest point of the lower branch of the $\eta=10$ equilibrium solution curve in Figure 1, and to the left of the upper branch of the $\eta=10$ equilibrium solution curve in Figure 2. In both cases, the comparison suggests that the representative solution given here is stable.

This illustrative solution assumes that the underlying disk is in the ambipolar conductivity regime. Ambipolar diffusion is likely a reasonable assumption in the outer regions of typical protostellar disks, but may not hold throughout the inner regions of protostellar disks, which are more likely in the Hall or Ohm conductivity regimes. However, it is worth noting in this connection that models of wind-driving disks have column densities in the inner regions that are much smaller than in typical turbulent-viscosity accretion disks. These smaller column densities suggest that the region of a wind-driving disk in which ambipolar diffusivity is a good approximation may be substantially larger than in other types of disk models.

\section{CONCLUSION}

The model for the global structure of a magnetized protostellar disk/wind system presented here improves on previous studies (e.g., WK93; L96; F97; OL01) by including, for the first time, a fully self-consistent treatment of flux migration on the accretion timescale. This treatment employs the approximation that the magnetic field on scales $z \lesssim r$ above the disk surface is very nearly potential, leading to a simple constraint on the surface field inclination (Equation (71) ). The model also improves on previous studies (e.g., SKW07) by extending the conductivity tensor formalism for describing non-ideal disk material from radially localized disk models to global, radially self-similar disk models.

The model presented here describes solutions for a disk and wind that are steady on timescales up to the flux migration timescale. Studies of simplified equilibrium models of disk winds by Lubow et al. (1994b), Cao \& Spruit (2002), and Campbell (2009) have raised the possibility that magnetocentrifugal disk winds are inherently unstable. Königl (2004, hereafter K04) showed that for strongly coupled wind-driving disk models of the sort described in WK93 there are two equilibrium solution branches, one stable and one unstable, and argued that protostellar systems correspond to the stable branch. This line of argument has received some support from simulations of axisymmetric, diffusive accretion disks launching self-collimating, magnetocentrifugal disk winds that have been carried out by Casse \& Keppens (2002, 2004) and Zanni et al. (2007). These simulations confirm that the resulting large-scale jets are continuous and steady over many dynamic timescales, providing support for the present model's quasi-steady state assumption. In turn, the semianalytic model presented here facilitates calculations that cannot currently be carried out in full numerical simulations.

The formulation presented here allows construction of global disk/wind models with realistic conductivity profiles in the disk. Finding matched disk/wind solutions of the sort presented here is a difficult task due to the imposed restrictions of requiring all angular mo- 
mentum transport to occur via the wind, and the strict self-similarity of the disk and wind solutions. In reality, protostellar disk/wind systems may transport angular momentum simultaneously through multiple mechanisms, such as magnetic braking, gravitational instability, and turbulence (see SKW07 for a radially localized disk model that includes angular momentum transport by both a wind and turbulence driven by the magnetorotational instability), and are unlikely to be globally selfsimilar. However, simple models of the sort presented here are valuable tools for investigating global processes in disk/wind systems, such as dust transport and processing (S. Teitler, in preparation). Studies of dust evolution processes in wind-driving disks will complement similar studies carried out in simple models of turbulent disks (e.g., Keller \& Gail 2004; Brauer et al. 2008) and in the X-wind model (e.g., Shu et al. 2001), and are of particular importance for wind-driving disks since small dust grains can have strong effects on the coupling of neutral disk material to magnetic fields (e.g., Nishi et al. 1991; Wardle 2007).

I thank Arieh Königl for his unwearying support and guidance over the past several years, and my thesis committee members Fausto Cattaneo, Fred Ciesla, Joshua Frieman, and Don York for their patience and advice. I also thank Raquel Salmeron, Konstantinos Tassis, and Mark Wardle for many helpful discussions. In addition, Raquel Salmeron and Zhi-Yun Li graciously made available unpublished material that proved very helpful. Finally, I thank the anonymous referee for many helpful comments. This work was supported in part by NASA Astrophysics Theory and Fundamental Physics Program grant NNX09AH38G and NSF grant AST-0908184.

\section{APPENDIX}

\section{CALCULATING $E_{R}^{\prime}$}

Given the values of the independent variable $s$ and the dependent variables $x, v_{r}, v_{\phi}, v_{z}, b_{r}, b_{\phi}, e_{r}$, and $\tilde{\rho}$, the auxiliary variables $b_{z}$ and $e_{\phi}$ can be obtained directly from the algebraic relations (32) and (46). Their derivatives are

$$
b_{z}^{\prime}=b_{r}^{\prime} s+b_{r}-2 \frac{x^{\prime}}{x^{3}}=b_{r}^{\prime} s-b_{r}
$$

and

$$
e_{\phi}^{\prime}=\left(\frac{1}{\zeta}-\frac{5}{2}\right) e_{\phi}(0) b_{r} x^{\frac{1}{\zeta}-\frac{1}{2}}
$$

The auxiliary variables $e_{\mathrm{c} r}$ and $e_{\mathrm{c} \phi}$ are obtained from Equation (39).

The next step is to combine the algebraic relations (37), (40), and (42) to obtain

$$
\begin{aligned}
\tilde{\sigma}_{\mathrm{O}} e_{\mathrm{c} z \|}+\frac{\tilde{\sigma}_{\mathrm{H}}}{b}\left(b_{r} e_{\mathrm{c} \phi \perp}-b_{\phi} e_{\mathrm{c} r \perp}\right)+\tilde{\sigma}_{\mathrm{P}} e_{\mathrm{c} z \perp}= & \left(\tilde{\sigma}_{\mathrm{O}} e_{\mathrm{c} r \|}+\frac{\tilde{\sigma}_{\mathrm{H}}}{b}\left(b_{\phi} e_{\mathrm{c} z \perp}-b_{z} e_{\mathrm{c} \phi \perp}\right)+\tilde{\sigma}_{\mathrm{P}} e_{\mathrm{c} r \perp}\right) s \\
& +\left(\frac{1}{\zeta}-1\right) \frac{b_{\phi}}{x} .
\end{aligned}
$$

Using Equations (43) and (44), we have

$$
\begin{array}{rr}
e_{\mathrm{c} r \|}=e_{\mathrm{c} r}-e_{\mathrm{c} r \perp} ; & e_{\mathrm{c} \phi \|}=\frac{b_{\phi}}{b_{r}}\left(e_{\mathrm{c} r}-e_{\mathrm{c} r \perp}\right) ; \\
e_{\mathrm{c} z \|}=\frac{b_{z}}{b_{r}}\left(e_{\mathrm{c} r}-e_{\mathrm{c} r \perp}\right) ; & e_{\mathrm{c} \phi \perp}=e_{\mathrm{c} \phi}-\frac{b_{\phi}}{b_{r}}\left(e_{\mathrm{c} r}-e_{\mathrm{c} r \perp}\right) ; \\
e_{\mathrm{c} z \perp}=-\frac{1}{b_{z}}\left(b_{r} e_{\mathrm{c} r \perp}+b_{\phi}\left[e_{\mathrm{c} \phi}-\frac{b_{\phi}}{b_{r}}\left(e_{\mathrm{c} r}-e_{\mathrm{c} r \perp}\right)\right]\right),
\end{array}
$$

giving $\boldsymbol{e}_{\mathrm{c} \|}$ and $\boldsymbol{e}_{\mathrm{c} \perp}$ in terms of $e_{\mathrm{c} r \perp}$ and the known quantities $e_{\mathrm{c} r}, e_{\mathrm{c} \phi}$, and $\boldsymbol{b}$. Substituting these expressions into Equation (A1) and solving, we obtain

$$
\begin{array}{r}
\left.e_{\mathrm{c} r \perp\left[\tilde{\sigma}_{\mathrm{O}}\left(s-\frac{b_{z}}{b_{r}}\right)-\right.} \tilde{\sigma}_{\mathrm{P}}\left(\frac{b_{r}}{b_{z}}+\frac{b_{\phi}^{2}}{b_{r} b_{z}}+s\right)+s \frac{\tilde{\sigma}_{\mathrm{H}}}{b}\left(\frac{b_{r}}{b_{\phi}} b_{z}+b_{\phi}^{3} b_{r} b_{z}+\frac{b_{\phi} b_{z}}{b_{z}}\right)\right]= \\
e_{\mathrm{c} r}\left[\tilde{\sigma}_{\mathrm{O}}\left(s-\frac{b_{z}}{b_{r}}\right)-\tilde{\sigma}_{\mathrm{P}} \frac{b_{\phi}^{2}}{b_{r} b_{z}}+\frac{\tilde{\sigma}_{\mathrm{H}}}{b}\left(b_{\phi}+s\left[\frac{b_{\phi}^{3}}{b_{r} b_{z}}+\frac{b_{\phi} b_{z}}{b_{r}}\right]\right)\right] \\
+e_{\mathrm{c} \phi}\left[\tilde{\sigma}_{\mathrm{P}} \frac{b_{\phi}}{b_{z}}-\frac{\tilde{\sigma}_{\mathrm{H}}}{b}\left(s\left[\frac{b_{\phi}^{2}}{b_{z}}+b_{z}\right]+b_{r}\right)\right]+\left(\frac{1}{\zeta}-1\right) \frac{b_{\phi}}{x}
\end{array}
$$


which simplifies to

$$
e_{\mathrm{c} r \perp}=A e_{\mathrm{cr} r}+B=A e_{r}+B+A\left(v_{r} b_{\phi}-v_{\phi} b_{r}\right),
$$

where

$$
A=\frac{\left[\tilde{\sigma}_{\mathrm{O}} b b_{z}+\tilde{\sigma}_{\mathrm{P}} x^{2} b b_{\phi}^{2}-s \tilde{\sigma}_{\mathrm{H}} x^{2} b^{2} b_{\phi}-\tilde{\sigma}_{\mathrm{H}} b_{r} b_{\phi}\right]}{\left[\left(\tilde{\sigma}_{\mathrm{O}}-\tilde{\sigma}_{\mathrm{P}}\right) b b_{z}+\tilde{\sigma}_{\mathrm{P}} x^{2} b^{3}-s \tilde{\sigma}_{\mathrm{H}} x^{2} b^{2} b_{\phi}\right]}
$$

and

$$
B=\frac{\left[e_{\mathrm{c} \phi}\left(-\tilde{\sigma}_{\mathrm{P}} x^{2} b b_{r} b_{\phi}+s \tilde{\sigma}_{\mathrm{H}} x^{2} b^{2} b_{r}+\tilde{\sigma}_{\mathrm{H}} b_{r}^{2}\right)-\left(\frac{1}{\zeta}-1\right) x b b_{r} b_{\phi} b_{z}\right]}{\left[\left(\tilde{\sigma}_{\mathrm{O}}-\tilde{\sigma}_{\mathrm{P}}\right) b b_{z}+\tilde{\sigma}_{\mathrm{P}} x^{2} b^{3}-s \tilde{\sigma}_{\mathrm{H}} x^{2} b^{2} b_{\phi}\right]} .
$$

Equation (A33) for $e_{\mathrm{c} r \perp}$ in terms of known quantities can be used to infer $e_{\mathrm{c} r \|}, e_{\mathrm{c} \phi \|}, e_{\mathrm{c} \phi \perp}, e_{\mathrm{c} z \|}, e_{\mathrm{c} z \perp}, e_{\mathrm{c} z}, e_{z}, j_{r}, j_{\phi}$, and $j_{z}$ using Equations (A2) and (401)-(42). These auxiliary quantities can in turn be used in Equations (48), (26)-(28), (36) and (33) to obtain $\tilde{\rho}^{\prime}, v_{r}^{\prime}, v_{\phi}^{\prime}, v_{z}^{\prime}, b_{r}^{\prime}$, and $b_{\phi}^{\prime}$.

The differential Equation (47) for $e_{r}^{\prime}$ involves the derivative of the auxiliary quantity $e_{z} ; e_{z}$ is given by

$$
\begin{aligned}
e_{z} & =e_{\mathrm{c} z}+v_{\phi} b_{r}-v_{r} b_{\phi} \\
& =e_{\mathrm{c} z \|}+e_{\mathrm{c} z \perp}+v_{\phi} b_{r}-v_{r} b_{\phi} \\
& =\frac{b_{z}}{b_{r}}\left(e_{\mathrm{c} r}-e_{\mathrm{c} r \perp}\right)-\frac{1}{b_{z}}\left(b_{r} e_{\mathrm{c} r \perp}+b_{\phi}\left[e_{\mathrm{c} \phi}-\frac{b_{\phi}}{b_{r}}\left(e_{\mathrm{c} r}-e_{\mathrm{c} r \perp}\right)\right]\right)+v_{\phi} b_{r}-v_{r} b_{\phi} .
\end{aligned}
$$

Upon substituting for $e_{\mathrm{c} r \perp}$ from Equation (A3), this becomes

$$
\begin{aligned}
e_{z}= & e_{r}\left(\frac{b_{z}}{b_{r}}[1-A]-\frac{b_{r}}{b_{z}} A+\frac{b_{\phi}^{2}}{b_{r} b_{z}}[1-A]\right) \\
& +\left(v_{\phi} b_{z}-v_{z} b_{\phi}\right)\left(\frac{b_{z}}{b_{r}}[1-A]-\frac{b_{r}}{b_{z}} A+\frac{b_{\phi}^{2}}{b_{r} b_{z}}[1-A]\right) \\
& -B \frac{b^{2}}{b_{r} b_{z}}-\frac{b_{\phi}}{b_{z}} e_{\mathrm{c} \phi}+v_{\phi} b_{r}-v_{r} b_{\phi} .
\end{aligned}
$$

Differentiating Equation (A4) for $e_{z}$ gives

$$
e_{z}^{\prime}=C e_{r}^{\prime}+D
$$

where

$$
\begin{aligned}
& C= \frac{b_{z}}{b_{r}}(1-A)-\frac{b_{r}}{b_{z}} A+\frac{b_{\phi}^{2}}{b_{r} b_{z}}(1-A)=\frac{b_{z}}{b_{r}}+\frac{b_{\phi}^{2}}{b_{r} b_{z}}-\frac{b^{2}}{b_{r} b_{z}} A \\
& D= C^{\prime} e_{r}+C\left(v_{\phi}^{\prime} b_{z}+v_{\phi} b_{z}^{\prime}-v_{z}^{\prime} b_{\phi}-v_{z} b_{\phi}^{\prime}\right)+C^{\prime}\left(v_{\phi} b_{z}-v_{z} b_{\phi}\right) \\
&-B^{\prime} \frac{b^{2}}{b_{r} b_{z}}-2 B \frac{b b^{\prime}}{b_{r} b_{z}}+B \frac{b^{2} b_{r}^{\prime}}{b_{r}^{2} b_{z}}+B \frac{b^{2} b_{z}^{\prime}}{b_{r} b_{z}^{2}}-\frac{b_{\phi}^{\prime}}{b_{z}} e_{\mathrm{c} \phi}+\frac{b_{\phi} b_{z}^{\prime}}{b_{z}^{2}} e_{\mathrm{c} \phi} \\
&-\frac{b_{\phi}}{b_{z}} e_{\mathrm{c} \phi}^{\prime}+\left(v_{\phi}^{\prime} b_{r}+v_{\phi} b_{r}^{\prime}-v_{r}^{\prime} b_{\phi}-v_{r} b_{\phi}^{\prime}\right) \\
& C^{\prime}=\frac{b_{z}^{\prime}}{b_{r}}-\frac{b_{z} b_{r}^{\prime}}{b_{r}^{2}}+2 \frac{b_{\phi} b_{\phi}^{\prime}}{b_{r} b_{z}}-\frac{b_{\phi}^{2} b_{r}^{\prime}}{b_{r}^{2} b_{z}}-\frac{b_{\phi}^{2} b_{z}^{\prime}}{b_{r} b_{z}^{2}}-2 \frac{b b^{\prime}}{b_{r} b_{z}} A+\frac{b^{2} b_{r}^{\prime}}{b_{r}^{2} b_{z}} A+\frac{b^{2} b_{z}^{\prime}}{b_{r} b_{z}^{2}} A-\frac{b^{2}}{b_{r} b_{z}} A^{\prime}
\end{aligned}
$$




$$
\begin{aligned}
A^{\prime}= & \left(\left[\tilde{\sigma}_{\mathrm{O}}^{\prime} b b_{z}+\tilde{\sigma}_{\mathrm{O}} b^{\prime} b_{z}+\tilde{\sigma}_{\mathrm{O}} b b_{z}^{\prime}+\tilde{\sigma}_{\mathrm{P}}^{\prime} x^{2} b b_{\phi}+2 \tilde{\sigma}_{\mathrm{P}} x x^{\prime} b b_{\phi}^{2}+\tilde{\sigma}_{\mathrm{P}} x^{2} b^{\prime} b_{\phi}^{2}+2 \tilde{\sigma}_{\mathrm{P}} x^{2} b b_{\phi} b_{\phi}^{\prime}\right.\right. \\
& -\tilde{\sigma}_{\mathrm{H}} x^{2} b^{2} b_{\phi}-s \tilde{\sigma}_{\mathrm{H}}^{\prime} x^{2} b^{2} b_{\phi}-2 s \tilde{\sigma}_{\mathrm{H}} x x^{\prime} b^{2} b_{\phi}^{2}-2 s \tilde{\sigma}_{\mathrm{H}} x^{2} b b^{\prime} b_{\phi}-s \tilde{\sigma}_{\mathrm{H}} x^{2} b^{2} b_{\phi}^{\prime} \\
& \left.\left.-\tilde{\sigma}_{\mathrm{H}}^{\prime} b_{r} b_{\phi}-\tilde{\sigma}_{\mathrm{H}} b_{r}^{\prime} b_{\phi}-\tilde{\sigma}_{\mathrm{H}} b_{r} b_{\phi}^{\prime}\right]\right) /\left[\left(\tilde{\sigma}_{\mathrm{O}}-\tilde{\sigma}_{\mathrm{P}}\right) b b_{z}+\tilde{\sigma}_{\mathrm{P}} x^{2} b^{3}-s \tilde{\sigma}_{\mathrm{H}} x^{2} b^{2} b_{\phi}\right] \\
& -\left([ \tilde { \sigma } _ { \mathrm { O } } b b _ { z } + \tilde { \sigma } _ { \mathrm { P } } x ^ { 2 } b b _ { \phi } ^ { 2 } - s \tilde { \sigma } _ { \mathrm { H } } x ^ { 2 } b ^ { 2 } b _ { \phi } - \tilde { \sigma } _ { \mathrm { H } } b _ { r } b _ { \phi } ] \left[\left(\tilde{\sigma}_{\mathrm{O}}^{\prime}-\tilde{\sigma}_{\mathrm{P}}^{\prime}\right) b b_{z}+\left(\tilde{\sigma}_{\mathrm{O}}-\tilde{\sigma}_{\mathrm{P}}\right) b^{\prime} b_{z}\right.\right. \\
& +\left(\tilde{\sigma}_{\mathrm{O}}-\tilde{\sigma}_{\mathrm{P}}\right) b b_{z}^{\prime}+\tilde{\sigma}_{\mathrm{P}} x^{2} b^{3}+2 \tilde{\sigma}_{\mathrm{P}} x x^{\prime} b^{3}+3 \tilde{\sigma}_{\mathrm{P}} x^{2} b^{2} b^{\prime}-\tilde{\sigma}_{\mathrm{H}} x^{2} b^{2} b_{\phi}-s \tilde{\sigma}_{\mathrm{H}}^{\prime} x^{2} b^{2} b_{\phi} \\
& \left.\left.-2 s \tilde{\sigma}_{\mathrm{H}} x x^{\prime} b^{2} b_{\phi}-2 s \tilde{\sigma}_{\mathrm{H}} x^{2} b b^{\prime} b_{\phi}-s \tilde{\sigma}_{\mathrm{H}} x^{2} b^{2} b_{\phi}^{\prime}\right]\right) /\left[\left(\tilde{\sigma}_{\mathrm{O}}-\tilde{\sigma}_{\mathrm{P}}\right) b b_{z}+\tilde{\sigma}_{\mathrm{P}} x^{2} b^{3}-s \tilde{\sigma}_{\mathrm{H}} x^{2} b^{2} b_{\phi}\right]^{2},
\end{aligned}
$$

and

$$
\begin{aligned}
B^{\prime}= & \left(\left[e_{\phi}^{\prime}+v_{z}^{\prime} b_{r}+v_{z} b_{r}^{\prime}-v_{r}^{\prime} b_{z}-v_{r} b_{z}^{\prime}\right]\left[-\tilde{\sigma}_{\mathrm{P}} x^{2} b b_{r} b_{\phi}+s \tilde{\sigma}_{\mathrm{H}} x^{2} b^{2} b_{r}+\tilde{\sigma}_{\mathrm{H}} b_{r}^{2}\right]\right. \\
& +e_{\mathrm{c} \phi}\left[-\tilde{\sigma}_{\mathrm{P}}^{\prime} x^{2} b b_{r} b_{\phi}-2 \tilde{\sigma}_{\mathrm{P}} x x^{\prime} b b_{r} b_{\phi}-\tilde{\sigma}_{\mathrm{P}} x^{2} b^{\prime} b_{r} b_{\phi}-\tilde{\sigma}_{\mathrm{P}} x^{2} b b_{r}^{\prime} b_{\phi}-\tilde{\sigma}_{\mathrm{P}} x^{2} b b_{r} b_{\phi}^{\prime}\right. \\
& +\tilde{\sigma}_{\mathrm{H}} x^{2} b^{2} b_{r}+s \tilde{\sigma}_{\mathrm{H}}^{\prime} x^{2} b^{2} b_{r}+2 s \tilde{\sigma}_{\mathrm{H}} x x^{\prime} b^{2} b_{r}+2 s \tilde{\sigma}_{\mathrm{H}} x^{2} b b^{\prime} b_{r}+s \tilde{\sigma}_{\mathrm{H}} x^{2} b^{2} b_{r}^{\prime} \\
& \left.+\tilde{\sigma}_{\mathrm{H}}^{\prime} b_{r}^{2}+2 \tilde{\sigma}_{\mathrm{H}} b_{r} b_{r}^{\prime}\right]-\left[\frac{1}{\zeta}-1\right]\left[x^{\prime} b b_{r} b_{\phi} b_{z}+x b^{\prime} b_{r} b_{\phi} b_{z}+x b b_{r}^{\prime} b_{\phi} b_{z}\right. \\
& \left.\left.+x b b_{r} b_{\phi}^{\prime} b_{z}+x b b_{r} b_{\phi} b_{z}^{\prime}\right]\right) /\left[\left(\tilde{\sigma}_{\mathrm{O}}-\tilde{\sigma}_{\mathrm{P}}\right) b b_{z}+\tilde{\sigma}_{\mathrm{P}} x^{2} b^{3}-s \tilde{\sigma}_{\mathrm{H}} x^{2} b^{2} b_{\phi}\right] \\
& -\left([ e _ { \mathrm { c } \phi } ( - \tilde { \sigma } _ { \mathrm { P } } x ^ { 2 } b b _ { r } b _ { \phi } + s \tilde { \sigma } _ { \mathrm { H } } x ^ { 2 } b ^ { 2 } b _ { r } + \tilde { \sigma } _ { \mathrm { H } } b _ { r } ^ { 2 } ) - ( \frac { 1 } { \zeta } - 1 ) x b b _ { r } b _ { \phi } b _ { z } ] \left[\left(\tilde{\sigma}_{\mathrm{O}}^{\prime}-\tilde{\sigma}_{\mathrm{P}}^{\prime}\right) b b_{z}\right.\right. \\
& +\left(\tilde{\sigma}_{\mathrm{O}}-\tilde{\sigma}_{\mathrm{P}}\right) b^{\prime} b_{z}+\left(\tilde{\sigma}_{\mathrm{O}}-\tilde{\sigma}_{\mathrm{P}}\right) b b_{z}^{\prime}+\tilde{\sigma}_{\mathrm{P}} x^{2} b^{3}+2 \tilde{\sigma}_{\mathrm{P}} x x^{\prime} b^{3}+3 \tilde{\sigma}_{\mathrm{P}} x^{2} b^{2} b^{\prime}-\tilde{\sigma}_{\mathrm{H}} x^{2} b^{2} b_{\phi}-s \tilde{\sigma}_{\mathrm{H}}^{\prime} x^{2} b^{2} b_{\phi} \\
& \left.\left.-2 s \tilde{\sigma}_{\mathrm{H}} x x^{\prime} b^{2} b_{\phi}-2 s \tilde{\sigma}_{\mathrm{H}} x^{2} b b^{\prime} b_{\phi}-s \tilde{\sigma}_{\mathrm{H}} x^{2} b^{2} b_{\phi}^{\prime}\right]\right) /\left[\left(\tilde{\sigma}_{\mathrm{O}}-\tilde{\sigma}_{\mathrm{P}}\right) b b_{z}+\tilde{\sigma}_{\mathrm{P}} x^{2} b^{3}-s \tilde{\sigma}_{\mathrm{H}} x^{2} b^{2} b_{\phi}\right]^{2} .
\end{aligned}
$$

Substituting Equation (A5) into Equation (47), we have

$$
e_{r}^{\prime}+s\left(C e_{r}^{\prime}+D\right)=\left(\frac{1}{\xi}-\frac{5}{2}\right)\left(\frac{x^{\prime}}{x}\left[e_{r}+e_{z} s\right]+e_{z}\right),
$$

which gives

$$
e_{r}^{\prime}=\frac{\left(\frac{1}{\xi}-\frac{5}{2}\right)\left(\frac{x^{\prime}}{x}\left[e_{r}+e_{z} s\right]+e_{z}\right)-D s}{1+C s}
$$




\section{TAYLOR SERIES EXPANSIONS FROM THE MIDPLANE}

As described in Section 2.1.3, the midplane is a singular point of the disk equations. The procedure adopted here for dealing with this issue is to calculate Taylor series expansions of the variables of integration from the midplane to a small distance $\Delta s=0.001$ above the midplane before beginning numerical integration of the disk equations. The Taylor series expansions for a general, non-ideal disk described using the conductivity tensor formalism are presented in Section B.1. The case of a disk in the ambipolar diffusion regime with constant neutral-field coupling parameter $\Upsilon$ is covered in Section B.2.

\section{General Case}

Differentiating Equation (31) once with respect to $s$, we have $x^{\prime \prime}=b_{r}^{\prime} x^{3}+3 b_{r} x^{2} x^{\prime}=b_{r}^{\prime} x^{3}+3 b_{r}^{2} x^{2}$; dropping terms that vanish at the midplane gives

$$
x_{0}^{\prime \prime}=b_{r, 0}^{\prime} .
$$

Differentiating Equation (48) once with respect to $s$ and dropping terms that vanish at the midplane gives

$$
\begin{aligned}
\tilde{\rho}_{0}^{\prime \prime}= & {\left[-\frac{1}{\theta}\right]\left[\left(2-\frac{2}{\zeta}\right) v_{r, 0}\left(v_{z, 0}^{\prime}-v_{r, 0}\right)+\left(4-\frac{2}{\zeta}\right) \theta\left(1+x_{0}^{\prime \prime}\right)\right.} \\
& \left.+v_{\phi, 0}^{2}+\nu\left(j_{\phi, 0}\left[b_{r, 0}^{\prime}+1\right]-b_{\phi, 0}^{\prime} j_{r, 0}\right)\right] .
\end{aligned}
$$

Differentiating Equation (47) once with respect to $s$ and dropping terms that vanish at the midplane gives

$$
e_{r, 0}^{\prime \prime}+e_{z, 0}^{\prime}=\left(\frac{1}{\xi}-\frac{5}{2}\right)\left(x_{0}^{\prime \prime} e_{r, 0}+e_{z, 0}^{\prime}\right)
$$

Differentiating the $z$ component of Equation (39) gives $e_{z, 0}^{\prime}=e_{\mathrm{c} z, 0}^{\prime}+v_{\phi, 0}^{\prime} b_{r, 0}^{\prime}-v_{r, 0} b_{\phi, 0}^{\prime}=e_{\mathrm{c} z \|, 0}^{\prime}+e_{\mathrm{c} z \perp, 0}^{\prime}+v_{\phi, 0}^{\prime} b_{r, 0}^{\prime}-v_{r, 0} b_{\phi, 0}^{\prime}$. Differentiating Equation (44) yields $e_{\mathrm{c} z \perp, 0}^{\prime}=-b_{r, 0}^{\prime} e_{\mathrm{c} r, 0}-b_{\phi, 0}^{\prime} e_{\mathrm{c} \phi, 0}$. Differentiating Equation (35) and Equation (42) gives $j_{z, 0}^{\prime}=(1 / \zeta-2) b_{\phi, 0}^{\prime}$ and $e_{\mathrm{c} z \|, 0}^{\prime}=\left(j_{z, 0}^{\prime}+\tilde{\sigma}_{\mathrm{H}, 0}\left[b_{\phi, 0}^{\prime} e_{\mathrm{c} r, 0}-b_{r, 0}^{\prime} e_{\mathrm{c} \phi, 0}\right]-\tilde{\sigma}_{\mathrm{P}, 0} e_{\mathrm{c} z \perp, 0}^{\prime}\right) / \tilde{\sigma}_{\mathrm{O}, 0}$, respectively. These relations can be used to solve for $e_{r, 0}^{\prime \prime}$ in Equation (B3).

Calculating $v_{r, 0}^{\prime \prime}$ and $v_{\phi, 0}^{\prime \prime}$ is somewhat more complex. Differentiating Equations (26) and (27) twice with respect to $s$ and dropping terms that vanish at the midplane gives

$$
\begin{aligned}
2\left(v_{z, 0}^{\prime}-v_{r, 0}\right) v_{r, 0}^{\prime \prime}-2 \theta \tilde{\rho}_{0}^{\prime \prime}= & v_{r, 0} v_{r, 0}^{\prime \prime}+2 v_{\phi, 0} v_{\phi, 0}^{\prime \prime}-v_{r, 0}\left(v_{z, 0}^{\prime}-v_{r, 0}\right) x_{0}^{\prime \prime}-3 \\
& +2\left(4-\frac{2}{\zeta}\right) \theta x_{0}^{\prime \prime}+\nu x_{0}^{\prime \prime} j_{\phi, 0}-\nu \tilde{\rho}_{0}^{\prime \prime} j_{\phi, 0}+\nu j_{\phi, 0}^{\prime \prime}-2 \nu j_{z, 0}^{\prime} b_{\phi}^{\prime}
\end{aligned}
$$

and

$$
\begin{aligned}
2\left(v_{z, 0}^{\prime}-v_{r, 0}\right) v_{\phi, 0}^{\prime \prime}= & -\frac{v_{r, 0}^{\prime \prime} v_{\phi, 0}}{2}-\frac{v_{r, 0} v_{\phi, 0}^{\prime \prime}}{2}+2 v_{\phi, 0} v_{\phi, 0}^{\prime \prime}-v_{\phi, 0}\left(v_{z, 0}^{\prime}-v_{r, 0}\right) x_{0}^{\prime \prime} \\
& -\nu x_{0}^{\prime \prime} j_{r, 0}+\nu \tilde{\rho}_{0}^{\prime \prime} j_{r, 0}-\nu j_{r, 0}^{\prime \prime}+2 \nu j_{z, 0}^{\prime} b_{r}^{\prime} .
\end{aligned}
$$

Expressions for the unknown quantities $j_{r, 0}^{\prime \prime}$ and $j_{\phi, 0}^{\prime \prime}$ can be derived by differentiating Equations (33) and (36) twice with respect to $s$ and dropping terms that vanish at the midplane, which gives

$$
\begin{aligned}
j_{r, 0}^{\prime \prime}= & \tilde{\sigma}_{\mathrm{O}, 0} e_{\mathrm{c} r \|, 0}^{\prime \prime}-\tilde{\sigma}_{\mathrm{H}, 0}^{\prime \prime} e_{\mathrm{c} \phi, 0}+\tilde{\sigma}_{\mathrm{H}, 0} b_{0}^{\prime \prime} e_{\mathrm{c} \phi, 0}+\tilde{\sigma}_{\mathrm{H}, 0}\left(2 b_{\phi, 0}^{\prime} e_{\mathrm{c} z \perp, 0}^{\prime}-e_{\mathrm{c} \phi \perp, 0}^{\prime \prime}\right) \\
& +\tilde{\sigma}_{\mathrm{P}, 0}^{\prime \prime} e_{\mathrm{c} r, 0}+\tilde{\sigma}_{\mathrm{P}, 0} e_{\mathrm{c} r \perp, 0}^{\prime \prime}
\end{aligned}
$$

and

$$
\begin{aligned}
j_{\phi, 0}^{\prime \prime}= & \tilde{\sigma}_{\mathrm{O}, 0} e_{\mathrm{c} \phi \|, 0}^{\prime \prime}+\tilde{\sigma}_{\mathrm{H}, 0}^{\prime \prime} e_{\mathrm{c} r, 0}-\tilde{\sigma}_{\mathrm{H}, 0} b_{0}^{\prime \prime} e_{\mathrm{c} r, 0}+\tilde{\sigma}_{\mathrm{H}, 0}\left(e_{\mathrm{c} r \perp, 0}^{\prime \prime}-2 b_{r, 0}^{\prime} e_{\mathrm{c} z \perp, 0}^{\prime}\right) \\
& +\tilde{\sigma}_{\mathrm{P}, 0}^{\prime \prime} e_{\mathrm{c} \phi, 0}+\tilde{\sigma}_{\mathrm{P}, 0} e_{\mathrm{c} \phi \perp, 0}^{\prime \prime} .
\end{aligned}
$$

We have $b_{0}^{\prime \prime}=b_{r, 0}^{\prime 2}+b_{\phi, 0}^{\prime 2}$, since $b_{z, 0}^{\prime}=b_{z, 0}^{\prime \prime}=0$. Differentiating Equation (43) twice gives the quantities $e_{\mathrm{c} r \|, 0}^{\prime \prime}$ and $e_{\mathrm{c} \phi \|, 0}^{\prime \prime}$ as $e_{\mathrm{c} r \|, 0}^{\prime \prime}=2 b_{r, 0}^{\prime} e_{\mathrm{c} z \|, 0}^{\prime}$ and $e_{\mathrm{c} \phi \|, 0}^{\prime \prime}=2 b_{\phi, 0}^{\prime} e_{\mathrm{c} z \|, 0}^{\prime}$. The quantities $e_{\mathrm{c} r \perp, 0}^{\prime \prime}$ and $e_{\mathrm{c} \phi \perp, 0}^{\prime \prime}$ are obtained by differentiating Equation (45) twice to obtain $e_{\mathrm{c} r \perp, 0}^{\prime \prime}=e_{\mathrm{c} r, 0}^{\prime \prime}-e_{\mathrm{c} r \|, 0}^{\prime \prime}$ and $e_{\mathrm{c} \phi \perp, 0}^{\prime \prime}=e_{\mathrm{c} \phi, 0}^{\prime \prime}-e_{\mathrm{c} \phi \|, 0}^{\prime \prime}$. Finally, differentiating Equation (39) twice gives $e_{c r, 0}^{\prime \prime}=e_{r, 0}^{\prime \prime}+v_{\phi, 0}^{\prime \prime}-2 v_{z, 0}^{\prime} b_{\phi, 0}^{\prime}$ and $e_{\mathrm{c} \phi, 0}^{\prime \prime}=2 v_{z, 0}^{\prime} b_{\phi, 0}^{\prime}-v_{r, 0}^{\prime \prime}$. Plugging these relations back into Equation (B4) and Equation (B5) gives two equations that can be solved for $v_{r, 0}^{\prime \prime}$ and $v_{\phi, 0}^{\prime \prime}$ in terms of known quantities. 


\section{Ambipolar Diffusion Case with Constant $\Upsilon$}

As in the general conductivity case, $x_{0}^{\prime \prime}=b_{r, 0}^{\prime}$.

Differentiating the relation $\boldsymbol{B} \cdot\left(\boldsymbol{V}_{\mathrm{i}}-\boldsymbol{V}\right) \stackrel{=}{=}$ once and evaluating at the midplane gives $v_{\mathrm{i} z, 0}^{\prime}=v_{z, 0}^{\prime}+$ $b_{r, 0}^{\prime}\left(v_{\mathrm{i} r, 0}-v_{r, 0}\right)+b_{\phi, 0}^{\prime}\left(v_{\mathrm{i} \phi, 0}-v_{\phi, 0}\right)$. Differentiating Equation (63) twice and evaluating at the midplane yields

$$
v_{\mathrm{i} r, 0}^{\prime \prime}=2 b_{r, 0}^{\prime} v_{\mathrm{i} z, 0}^{\prime}-\left(\frac{1}{\zeta}-\frac{5}{2}\right) \epsilon_{\mathrm{B}} \theta^{\frac{1}{2}} b_{r, 0}^{\prime} .
$$

Differentiating the $z$ component of Equation (8) (cf. Equation (12) of L96) once and evaluating at the midplane gives

$$
\tilde{\rho}_{0}^{\prime \prime}=\left(v_{z, 0}^{\prime}\left[\frac{3 v_{r, 0}}{2}-v_{z, 0}^{\prime}\right]-\left[4-\frac{2}{\zeta}\right] \theta x_{0}^{\prime \prime}-1+\Upsilon_{0}\left[v_{\mathrm{i} z, 0}^{\prime}-v_{z, 0}^{\prime}\right]\right) / \theta
$$

Differentiating Equation (64) three times and evaluating at the midplane yields

$$
v_{\mathrm{i} \phi, 0}^{\prime \prime}=\frac{b_{\phi, 0}^{\prime}}{b_{r, 0}^{\prime}} v_{\mathrm{i} r, 0}^{\prime \prime}+\left(v_{\mathrm{i} \phi, 0} b_{r, 0}^{\prime}-v_{\mathrm{i} r, 0} b_{\phi, 0}^{\prime}\right) .
$$

Finally, differentiating the $r$ and $\phi$ components of Equation (8) (cf. Equations (13) and (14) of L96) twice and evaluating at the midplane gives

$$
\begin{aligned}
\left(3 v_{r, 0}-2 v_{z, 0}^{\prime}-\Upsilon_{0}\right) v_{r, 0}^{\prime \prime}+2 v_{\phi, 0} v_{\phi, 0}^{\prime \prime}= & v_{r, 0}\left(v_{z, 0}^{\prime}-v_{r, 0}\right) x_{0}^{\prime \prime}-2 \theta \tilde{\rho}_{0}^{\prime \prime}-2\left(4-\frac{2}{\zeta}\right) \theta x_{0}^{\prime \prime} \\
& -x_{0}^{\prime \prime}+3-\Upsilon_{0} v_{\mathrm{i} r, 0}^{\prime \prime}+\Upsilon_{0}^{\prime \prime}\left(v_{r, 0}^{\prime \prime}-v_{\mathrm{i} r, 0}^{\prime \prime}\right)
\end{aligned}
$$

and

$$
\frac{v_{\phi, 0}}{2} v_{r, 0}^{\prime \prime}+\left(2 v_{z, 0}^{\prime \prime}-\frac{3}{2} v_{r, 0}+\Upsilon_{0}\right) v_{\phi, 0}^{\prime \prime}=\Upsilon_{0} v_{\mathrm{i} \phi, 0}^{\prime \prime}+\Upsilon_{0}^{\prime \prime}\left(v_{\mathrm{i} \phi, 0}-v_{\phi, 0}\right)-v_{\phi, 0}\left(v_{z, 0}^{\prime}-v_{r, 0}\right) x_{0}^{\prime \prime} .
$$

These equations can be solved for $v_{r, 0}^{\prime \prime}$ and $v_{\phi, 0}^{\prime \prime}$ in terms of known quantities. 


\section{REFERENCES}

Andrews, S. M. \& Williams, J. P. 2007, ApJ, 659, 705

Balbus, S. A., \& Hawley, J. F. 1991, ApJ, 376, 214

Bally, J., Reipurth, B., \& Davis, C. J. 2007, in Protostars \& Planets V, ed. B. Reipurth, D. Jewitt, \& K. Keil (Tucson: Univ. Arizona Press), 215

Blandford, R. D., \& Payne, D. G. 1982, MNRAS, 199, 883 (BP82) Brauer, F., Dullemond, C. P., \& Henning, Th. 2008, A\&A 480, 859

Campbell, C. G. 2003, MNRAS, 345, 123

Campbell, C. G. 2005, MNRAS, 361, 396

Campbell, C. G. 2009, MNRAS, 392, 271

Cao, X., \& Spruit, H. C. 2002, A\&A, 385, 289

Casse, F., \& Ferreira, J. 2000, A\&A, 361, 1178

Casse, F., \& Keppens, R. 2002, ApJ, 581, 988

Casse, F., \& Keppens, R. 2004, ApJ, 601, 90

Ciolek, G. E., \& Mouschovias, T. Ch. 1993, ApJ, 418, 774

Draine, B. T., Roberge, W. G., \& Dalgarno, A. 1983, ApJ, 264, 485

Ferreira, J. 1997, A\&A, 319, 340 (F97)

Ferreira, J., \& Casse, F. 2004, ApJ, 601, L139

Guan, X., \& Gammie, C. F. 2009, ApJ, 697, 1901

Hartigan, P., Edwards, S., \& Ghandour, L. 1995, ApJ, 452, 736

Hayashi, C. 1981, Prog Theor Phys Supp, 70, 35

Heyvaerts, J., \& Norman, C. 1989, ApJ, 347, 1055

Keller, Ch., \& Gail, H.-P. 2004, A\&A, 415, 1177

Königl, A. 1989, ApJ, 342, 208

Königl, A. 2004, ApJ, 617, 1267 (K04)

Königl, A., \& Salmeron, R. 2011, in Physical Processes in Circumstellar Disks around Young Stars, ed. P. J. V. Garcia (Chicago: Univ. Chicago Press), in press (arXiv:1004:1875)
Königl, A., Salmeron, R \& Wardle, M. 2010, MNRAS, 401, 479 Lesur, G., \& Longaretti, P.-Y. 2009, A\&A, 504, 309

Levy, E. H., \& Sonett, C. P. 1978, in Protostars \& Planets, ed. T. Gehrels (Tucson: Univ. Arizona Press), 516

Li, Z.-Y. 1995, ApJ, 444, 848

Li, Z.-Y. 1996, ApJ, 465, 855 (L96)

Lovelace, R. V. E., Rothstein, D. M., \& Bisnovatyi-Kogan, G. S. 2009, ApJ, 701, 885

Lubow, S. H., Papaloizou, J. C. B., \& Pringle J. E. 1994, MNRAS, 267, 235

Lubow, S. H., Papaloizou, J. C. B., \& Pringle J. E. 1994, MNRAS, 268, 1010

Nishi, R., Nakano, T., \& Umebayashi, T. 1991, ApJ, 368, 181

Ogilvie, G. I. \& Livio, M. 2001, ApJ, 553, 158 (OL01)

Ouyed, R., \& Pudritz, R. E. 1997, ApJ, 482, 712

Rothstein, D. M., \& Lovelace, R. V. E. 2008, ApJ, 677, 1221

Salmeron, R., Königl, A., \& Wardle, M. 2007, MNRAS, 375, 177 (SKW07)

Salmeron, R., Königl, A., \& Wardle, M. 2011, MNRAS, in press (arXiv:1006.0299) (SKW11)

Salmeron, R., \& Wardle, M. 2005, MNRAS, 361, 45

Shu, F. H., Shang, H., Gounelle, M., Glassgold, A. E., \& Lee, T. 2001, ApJ, 548, 1029

Vlahakis, N., Tsinganos, K., Sauty, C., \& Trussoni, E. 2000, MNRAS, 318, 417

Wardle, M. 2007, Ap\&SS, 311, 35

Wardle, M., \& Königl, A. 1993, ApJ, 410, 218 (WK93)

Zanni, C., Ferrari, A., Rosner, R., Bodo, G., \& Massaglia, S. 2007, A'\&A, 469, 811 